\title{
Evolutionary Contingency as Non-Trivial Objective Probability: Biological Evitability and Evolutionary Trajectories
}

\author{
Author: T.Y. William Wong \\ Affiliation: Department of History and Philosophy of Science, University of Cambridge
}

Address: Department of History and Philosophy of Science, University of Cambridge

Free School Lane, Cambridge

United Kingdom

CB2 3RH

E-mail: tyww2@cam.ac.uk

ORCID: 0000-0002-2750-7990

\begin{abstract}
:
Contingency-theorists have put forth differing accounts of evolutionary contingency. The bulk of these accounts abstractly refer to certain causal structures in which an evolutionarily contingent outcome is supposedly embedded. For example, an outcome is evolutionarily contingent if it is at the end of a 'pathdependent' or 'causally dependent' causal chain. However, this paper argues that many of these proposals fail to include a desideratum - the notion of biological evitability or that evolutionary outcomes could have been otherwise - that for good theoretical reasons ought to be part of an account of evolutionary contingency. Although an inclusion of this desideratum might seem obvious enough, under some existing accounts, an outcome can be contingent yet inevitable all the same. In my diagnosis of this issue, I develop the idea of trajectory propensity to highlight the fact that there are plausible biological scenarios in which causal structures, alone, fail to exhaustively determine the biological evitability of evolutionary forms. In the second half of the paper, I present two additional desiderata of an account of evolutionary contingency and, subsequently, proffer a novel account of evolutionary contingency as non-trivial objective probability which overcomes the shortcomings of some previous proposals. According to this outcome-based account, contingency claims are probabilistic statements about an evolutionary outcome's objective probability of evolution within a specifically defined modal range: an outcome, $\mathrm{O}$, is evolutionarily contingent in modal range, $\mathrm{R}$, to the degree of objective probability, $\mathrm{P}$ (where $\mathrm{P}$ is in between 1 and 0 ).

Keywords: Evolutionary Contingency, Modal Range, Biological Evitability, Evolutionary Trajectories, Trajectory Propensity, Counterfactual Robustness
\end{abstract}

\section{Acknowledgements:}

I would like to thank Tyler Brunet, Stijn Conix, Adrian Currie, Marta Halina, Alistair Isaac, Tim Lewens, Alison McConwell, Jacob Stegenga, Pablo Zendejas Medina, and the anonymous referees for their comments on some iteration of this paper. 


\section{Evolutionary Contingency as Non-Trivial Objective Probability: Biological Evitability and Evolutionary Trajectories}

\section{Highlights}

- There is no present consensus as to what evolutionary contingency amounts to. This paper's chief concern is the proper characterisation of evolutionary contingency.

- A number of recent proposals of contingency are inadequate in various respects. Most concerning is that, contrary to good theory, some of these accounts permit an outcome to be evolutionarily contingent yet inevitable, all the same (under certain demonstrable conditions).

- The paper develops a dynamical systems analysis to highlight two forms of modalities that have been implicit in past discussions of evolutionary contingency. Whilst counterfactual robustness has received much attention, many previous proposals of contingency overlook the form of modality that I call trajectory propensity, which makes explicit the fact that evolutionary outcomes are often preceded by a series of antecedents that, themselves, have a dlow objective probability of occurrence.

- Alongside a presentation of some desiderata of an account of contingency, the paper puts forth a novel account of evolutionary contingency as non-trivial objective probability that overcomes the shortcomings of some previous proposals. According to this account, an outcome, $\mathrm{O}$, is evolutionarily contingent in modal range, $\mathrm{R}$, to the degree of objective probability, $\mathrm{P}$ (where $\mathrm{P}$ is in between 1 and 0 ). 


\section{Evolutionary Contingency as Non-Trivial Objective Probability: Biological Evitability and Evolutionary Trajectories}

\section{Introduction}

Almost all textbooks in evolutionary biology, nowadays, seem to banally commend the diversity and/or disparity of biological forms found in the natural world. These textbooks allude to the myriad of clever solutions to ecological problems and revel in the supposed power of evolution to conjure up forms that anthropocentrically seem impressive to us. But considering all of this remarkability, how much of it was inevitable in its evolution? Or were they, human forms included, merely historical accidents? These questions, I take it, are at the heart of the idea of evolutionary contingency.

Although this might seem obvious enough, of the various senses of evolutionary contingency proposed in the literature, most characterisations (save three) fail to entail the possibility of alternative outcomes. In fact, under some accounts of evolutionary contingency, an outcome can be evolutionarily contingent yet inevitable, all the same. This is significant for such a result does not gel well with our intuitions nor, as we shall see, with good theory - it indicates that there is something amiss with these accounts.

Contingency-theorists have presented a wide range of different and, sometimes, competing accounts of evolutionary contingency. They abstractly refer to a variety of etiological structures with a particular set of causal conditions (e.g. 'causal dependence', 'path dependence', 'sensitivity to initial conditions'), in which evolutionarily contingent outcomes are supposedly embedded. For example, an outcome is taken to be evolutionarily contingent if it is an outcome at the end of a path dependent causal chain, or if it is an outcome that is sensitive to initial conditions.

The objective of the present paper is to consider the candidacy of some recent proposals of contingency in light of three desiderata and, then, to proffer a novel account for consideration. As alluded to earlier, the most significant shortcoming of some previous proposals is that the causal conditions outlined by these accounts can fall short of entailing a special kind of modal profile - i.e. biological evitability - for evolutionary outcomes. However, the biological evitability of evolutionary outcomes, or that evolutionary outcomes could have been otherwise in the face of a set of nomologies and histories, is a desideratum of an account of evolutionary contingency since without such a feature, contingency claims are no longer what they are ordinarily taken to be. Moreover, without biological evitability, some of the processes putatively taken to account for evolutionary contingency (e.g. 'fluctuating adaptive landscapes', 'global system changes') are theoretically excluded.

The failure of certain senses of contingency to entail the evitability of outcomes is owed to the fact that there has been a narrow focus on the etiological dynamics of (a set of) evolutionary trajectories within an evolutionary system (e.g. whether two or more causal chains with similar starting points lead to markedly different outcomes), and a consequent failure to recognise the distinct probability of remaining on a trajectory (what I shall later call 'trajectory propensity') within an evolutionary system. There are plausible biological scenarios where a high sensitivity to initial conditions, for example, is coupled with high trajectory propensity such that the evolutionary outcome was, after all, inevitable (or close to it). Ultimately, I conclude that evolutionary contingency ought to be sure to assert the right modal profile (i.e. biological evitability) for outcomes by being attuned to the possibility of high trajectory propensity rather than merely asserting certain etiological structures in which they are found. 
However, in addition to biological evitability, there are other desiderata for an account of evolutionary contingency. In this paper, I identify and present two additional desiderata. For example, it was a central theme of Gould's $(1989,2002)$ overarching project to emphasise the importance of history for evolutionary events, and contingency was (in a particular Gouldian way) to be the vessel by which that is true. Contingency-theorists have continued along this avenue of thought (e.g. Beatty and Desjardins, 2009; Beatty and Carrera, 2011; Desjardins, 2011a, 2011b, 2016) though, as we shall see, there is not just one way in which history can be important. Another often-cited desideratum is that evolutionary contingency ought to be able to vary in degree (e.g. Beatty, 1995, 2006; Desjardins, 2011a; Turner, 2011a; Powell, 2012). By that, it is minimally meant that whatever contingency is, there ought to be a spectrum of views ranging from the extreme to the intermediate. However, the way in which contingency varies in degree is very much dependent on the sense of contingency invoked. In fact, it is even non-sensical under some accounts. The need for a concept that varies in degree is supported by the putative fact that various levels of contingency are seemingly exhibited across distinct evolutionary domains, and that a proper account of contingency ought to be able to track such heterogeneity.

Alongside my presentation of the three desiderata ${ }^{1}$, I shall proffer an original account of evolutionary contingency. I submit that evolutionary contingency can be understood as asserting the non-trivial (not one or zero) objective probability of an evolutionary outcome within a specified range of evolutionary scenarios (modal range). Although such a solely probabilistic account differs from previous etiological and/or structural proposals of contingency, it is not out of place within evolutionary biology. After all, modern evolutionary theory is an inherently probabilistic enterprise in that it makes use of both subjective and objective probabilities in concepts such as fitness, selection coefficients, the law of segregation, and genetic drift. Contingency claims are no different and are merely assertations about the objective probability that certain outcomes evolve which, considering that they are under the purview of certain biological nomologies and histories, present as interesting scientific propositions.

This paper is divided into two halves: a negative element, criticising existing senses of contingency, followed by a more constructive element proposing the novel account. The plan is as follows: I begin with a brief primer on Beatty's (2006) two senses of evolutionary contingency. In section 2, I then unpack the idea of biological evitability and discuss its importance: it appears that previous usages of evolutionary contingency have implicitly subsumed the feature of biological evitability. This is no surprise as Gould, himself, consistently presented evolutionary contingency as if it implied alternative possibilities of evolutionary outcomes. After having motivated the thought that a theoretically sound notion of evolutionary contingency entails evitability, I argue that Beatty's (2006) causal dependence sense of contingency fails to do so. In section 3, I formally develop the idea of trajectory propensity, in a dynamical systems analysis, in order to highlight two forms of modalities at play within evolutionary systems. With this new theoretical machinery, I demonstrate that other senses of evolutionary contingency such as sensitivity to initial conditions and a specific understanding of path dependence also fail to entail evitability. In the latter half, I identify two additional desiderata and offer a novel account of evolutionary contingency as non-trivial objective probability that is able satisfy all three desiderata. However, Desjardins' path dependence account

\footnotetext{
${ }^{1} \mathrm{~A}$ brief note at this point: there can, in principle, be more than three desiderata for an account of evolutionary contingency (candidates include: empirical tractability, parsimony, and, (in)determinism neutrality). I present the three desiderata to evaluate the new account as a feasible alternative. See section 5 for further discussion.
} 
fares equally as well with respect to the desiderata and so, it is not clear that there is one superior account that ought to be understood as evolutionary contingency. Barring additional means for resolution, I advocate a sense of pluralism for evolutionary contingency. I conclude the paper by sketching some of the ways that contingency claims under the new account can be empirically evaluated.

\section{Evolutionary Contingency à la Beatty}

In Wonderful Life: The Burgess Shale and the Nature of History (1989), Gould proposed a thought experiment that neatly captures the central idea behind evolutionary contingency²:

I call this experiment "replaying life's tape." You press the rewind button and, making sure you thoroughly erase everything that actually happened, go back to any time and place in the past... Then let the tape run again and see if the repetition looks at all like the original. (Gould 1989, pg. 48)

Gould's expectation was that the outcome would be different from the original. However, he seems to speak of different versions of the tape of life. In some instances, Gould suggests replaying the tape of life from the same initial conditions:

We can explain an event after it occurs, but contingency precludes its repetition, even from an identical starting point (Gould, 1989, pg. 278)

Replay the tape a million times from a Burgess beginning... (Gould, 1989, pg. 286)

...I agree that the most fascinating aspects of life on earth is that it would probably play itself out in a totally different way if we started again from the same initial conditions (Letter from Gould to Schopf, 25 June 1979)

But, elsewhere, Gould suggests replaying the tape of life from different initial conditions:

Any replay, altered by an apparently insignificant jot or tittle at the outset, would have yielded an equally sensible and resolvable outcome of entirely different form (Gould, 1989, pg. 289)

Alter any early event, ever so slightly and without apparent importance at the time, and evolution cascades into a radically different channel (Gould, 1989, pg. 51)

It may be the case that Gould did not think that this amounted to any important difference and thus, glossed over the specificity of the point of rewind. However, Beatty (2006) recognises that the two versions of the tape of life metaphor amount to two importantly different senses of evolutionary contingency: that which he calls the 'unpredictability sense' and the 'causal dependence sense'.

Beatty's (2006) unpredictability sense of evolutionary contingency asserts that different evolutionary outcomes could result from the same starting points or "initially indistinguishable ancestral environments" ${ }^{3}$ (Ibid.). Even if the tape of life were replayed from the same initial conditions,

\footnotetext{
${ }^{2}$ Whilst the tape of life metaphor is most popularly known from Wonderful Life (1989), Gould has been recently been accused (e.g. Baron, 2011; Erwin, 2016) of "borrowing" the metaphor from Conway Morris (1985). However, this is mistaken as Gould has been employing the metaphor prior to Conway Morris publicly in 1976 (i.e. Gould, 1976) and, furthermore, privately in a letter to Schopf (Gould, 1979). The tape of life faithfully originated with Gould.

${ }^{3}$ Turner (2011a) notes that the term 'unpredictability' is infelicitous for it might be inappropriately suggestive of an epistemic dimension. One ought to remember that, despite the ample use of epistemic terms in the
} 
different outcomes could result. Put in another way, the initial conditions are causally insufficient to guarantee the same result.

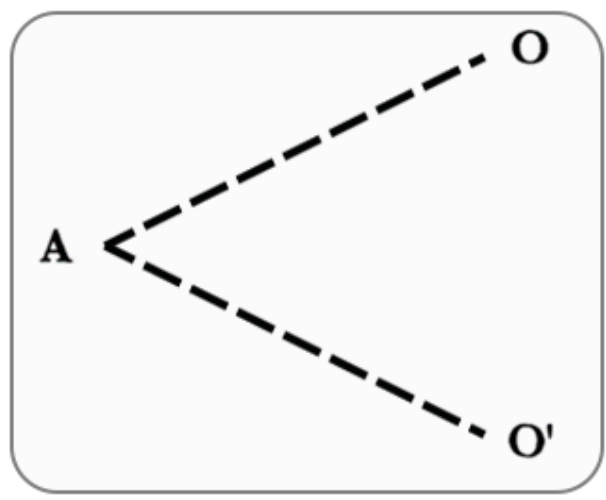

Figure 1. Unpredictability Sense: Ancestral state A could result in O or O'.

Now, at first glance, this seems to be committed to indeterminism since it suggests that the same initial conditions could result in different outcomes whilst determinism asserts just that the state of the universe at any given time, along with the natural laws, uniquely determines the subsequent states of the universe (Earman, 1986) ${ }^{4}$. On this issue, Beatty (2006) denies that the unpredictability sense is tantamount to indeterminism by appealing to the possibility of divergences in the evolutionary path due to what he calls the 'sources of contingency' - phenomena like random mutation, mutational ordering, genetic drift, and more. These sources of contingency are supposed to lack counterfactual robustness as to occur differently upon replays of the tape of life such that you could very well have, for example, different mutations or drift outcomes upon each replay. So, although the initial conditions may be the same, 'sources of contingency' will direct evolution onto different trajectories, ultimately resulting in different outcomes ${ }^{5}$.

The sense of contingency associated with the second version of the tape of life is what Beatty calls the 'causal dependence' sense. According to this sense, it is not that the same antecedent events lead to different outcomes (and hence, there is no longer a tension with determinism); rather outcomes are causally dependent, in an almost trivial way, on past events such that history is said to matter. The occurrence of certain antecedent events is, in Beatty's (2006) words, "necessary" or "strongly necessary" for certain evolutionary outcomes. But, quite crucially, it is not just any type of necessity at play; more specifically, what Beatty is referring to is causal necessity (not metaphysical necessity). That is - if $A$ is causally necessary for $O$, then $O$ could not have occurred without $A$. This, alone, would be akin to simple causal necessity as it is ordinarily understood in the philosophical literature.

contingency literature (especially, Gould's writings), evolutionary contingency is not about what epistemic agents can say about evolution, but about how evolution actually behaves lest evolutionary contingency devolve into something less interesting.

${ }^{4}$ This seems to present a two-horned dilemma: (i) either eschew determinism in favour of the unpredictability sense or, (ii) vice versa. However, I doubt that the former option will be readily adopted by biologists and philosophers given their pervasive fears of abandoning determinism (though Beatty, 2016, 2017 are exceptions).

${ }^{5}$ It is still somewhat of a mystery as to why these sources of contingency would not just yield the same results repeatedly. Due to lack of space, I cannot say any further about possible solutions to the indeterminism issue though see Millstein (2011) and List \& Pivato (2015) for what, I think, could be developed into a 'hidden variables' or 'coarse-graining function' solution. For now, I must 'black-box' the (in)determinism issue. 
But, charitably interpreted, there must be a subtle, additional element to Beatty's causal dependence as it relates to evolutionary contingency. To this end, Beatty (2006) additionally states that: had the antecedent events not occurred or occurred differently, then the evolutionary outcome would not have occurred or would have occurred differently. Strictly speaking, the second element regarding 'differential antecedents to differential outcomes' does not logically follow from the simple causal necessity of an antecedent. Rather, support for this second element seems to stem entirely from the following passage in Wonderful Life:

Historical explanations take the form of narrative: $E$, the phenomenon to be explained, arose because $D$ came before, preceded by $C, B$, and A. If any of these earlier stages had not occurred, or had transpired in a different way, then $\mathrm{E}$ would not exist (or would be present in a substantially altered form, E, requiring a different explanation). I am not speaking of randomness... but of the central principle of all history - contingency (Gould, 1989, pg. 283)

Thus, Beatty (2006) reasons that if evolutionary outcomes are 'causally dependent' on the past, then replaying the tape of life from even slightly different antecedent conditions would result in different outcomes. Once the second element is incorporated, Beatty's causal dependence appears to be similar to 'sensitivity to initial conditions' - a subtly different sense of contingency that we will soon encounter. Nonetheless, the second element is crucial, for it lends the causal dependence sense a historical element. Since any future outcome would be necessarily associated with a particular antecedent event, any future would be inscribed with the "inerasable signatures" (Gould, 1989, pg. 283) of the past. For this reason, the causal dependence sense bodes well for Gould's concomitant project of emphasising the importance of history (a desideratum that I will later discuss). But the replay experiment was meant to illustrate more than merely the importance of history; it was meant to also portray a shaky, uncertain future - or a possible difference in outcome. The problem, as I shall show in the subsequent section, is that the causal dependence sense, on its own, does not entail such a result.

\section{Biological Evitability: Evolutionary Forms Could Have Been Otherwise}

Consider the intuitive idea that contingent events are those that could have been otherwise. Borrowing some theoretical apparatus from standard metaphysics: if an event is contingent, then it occurs in the actual world, but there is at least one possible world in which the event does not occur. Conversely, if an event is necessary, then the event occurs in all possible worlds. Notice that this concept can be made to vary in degree: an event is more contingent just in case there are fewer possible worlds in which it occurs; or, if one prefers, an event is more contingent just in case some possible, non-actual, world where the event does not hold is closer (Lewis, 1973, 1986) to the actual world than any possible where the event does hold ${ }^{6}$.

Similarly, one may wonder whether evolutionary outcomes could have been otherwise. Are the evolutionary outcomes we see today - from the birds and the bats to the worms that burrow in our earth - occurrences in all evolutionary environments (the appropriate container as opposed to

\footnotetext{
${ }^{6}$ There are infinite possible worlds and so, there is a 'counting' problem. In lieu of counting, worlds can be ordered according to Lewis's $(1973,1986)$ comparative similarity or closeness relation. But one can also 'count' the number of possible worlds (despite there being an infinite number of them) by some 'external measure' like a probability measure. Plausibly, Evitability Measure (Probability): A biological form A is evitable to the degree $x$ iff the objective chance of the occurrence of biological form $A$ is 1-x. The probability measure seems superior to a Lewisian closeness measure since it outputs degrees of evitability instead of contrast-classed propositions. All of this, however, is to foreshadow what is to come - i.e. the probabilistic account of contingency.
} 
worlds) across some to be defined range of environments? Or is there at least one evolutionary environment (in the range) in which those species do not evolve (or evolve differently)?

Biological Evitability: A biological form is evitable if and only if there is an evolutionary scenario in some range of environments (i.e. the modal range) in which it does not evolve.

First and foremost, this is a biological notion rather than a metaphysical notion since evitability naturally depends on the biological laws of the range of evolutionary environments under consideration. If the relevant biological nomologies had been different, then the evitability of a form is likely to be different. But notice that under this definition, there has been no restriction on the extent of modality or the modal range at play (c.f. Wong, 2019). There ought to be some means of modal circumscription. I shall return to the idea of the modal range in section 5 .

As for biological evitability, unlike philosophical discussions in metaphysics about 'contingent worlds', there seems to exist a prevalent explanatory strategy inscribed in the evolutionary modern synthesis that would quite conveniently account for the inevitability (or close to inevitability) of biological outcomes across evolutionary environments. That is - at first glance, there seems to be biological reasons for thinking that the same outcomes would occur across all evolutionary environments for the engine of natural selection would push towards a very specific set of globally optimal forms ${ }^{7}$. In other words, insofar as there are a limited number of optimal forms and natural selection-cum-mutation is sufficient to result in optimal forms, then the same forms would evolve time and time again on account of their optimality.

On the other hand, opponents of this kind of thinking ('internalists' like Gould) advocate the presence of chancy historical and/or phylogenetic constraints that limit the power of natural selection. They claim that due to the influence of historical events in circumscribing the ancestral genetic material, the right sort of variations required for the evolution of the globally optimal forms may not be available. As such, the globally optimal forms may not be repeatedly reached in all the evolutionary environments; whichever forms are reached, in an evolutionary scenario, is dependent on the history and phylogenetics of that evolutionary scenario ${ }^{8}$.

It is within such dichotomous narratives (i.e. externalism vs. internalism) that contingency-theorists have frequently forged a link between the inevitability of forms and adaptationism (Beatty, 2006; Powell, 2012; Turner, 2011b). There is a putative connection between the biological inevitability of forms and adaptationism. If the inevitability of evolutionary outcomes is false, then it seems to say the following about the power of natural selection: natural selection has failed to push towards the same forms in phenotypic space. After all, if natural selection were truly all-powerful, then the globally optimal forms would be inevitable. However, the link between biological inevitability and adaptationism can be severed insofar as any one of many assumptions are false (inter alia): (i) there is a limited number of optimal forms (there are not multiple equally-as-adaptive solutions to the same selection problem), (ii) the power of natural selection includes the capacity to generate variations, (iii) the adaptive landscape (and hence, globally optimal forms) is counterfactually robust,

\footnotetext{
${ }^{7}$ There are two major assumptions: the first assumption is that the globally optimal forms are universal across environments. The second assumption is that there are a limited number of optimal solutions to an ecological/selective problem (c.f. Wong, 2019)

${ }^{8}$ Constraints (internal or external) are like a double-edged sword. They can be invoked to explain the evitability of forms as in the Gouldian narrative by appealing to chancy antecedent events (e.g. phylogenetic events). However, they can also be invoked to support a non-contingent view of life: constraints (especially, functional ones imposed by the laws of nature) would set supposedly universal boundaries so tight that, time and time again, only certain forms would arise.
} 
(iv) natural selection, itself, is not dependent on contingent events (see McConwell and Currie, 2016). Nonetheless, the degree to which the biological (in)evitability of forms is informative for adaptationism is dependent on how likely these assumptions are true; in other words, the link is conditional. And, perhaps, depending on which assumptions fail, evitability can be telling of which flavour of adaptationism to adopt.

The biological evitability of biological forms is also relevant to Gould's discussion of the Burgess Shale. Wonderful Life (1989) argued that the re-interpretation of the Burgess Shale (by Whittington and his two graduate students which, ironically, included one of Gould's opponents: Conway Morris) showed that there was greatest disparity during the Cambrian period. This disparity was exhibited by the plethora of 'wonderful' species, each with vastly different body plans. However, as Gould argues, the Cambrian was wrought by a series of lottery-like, fitness-insensitive mass extinctions whereby only a few survivors remained ${ }^{9}$. However, importantly, it was one of the surviving species - the Pikaia - that, then, became the ancestor to all modern vertebrates (according to Gould's account). Gould's crucial point was that it could have been any other species that survived rather than the Pikaia - perhaps, one containing a radically disparate body plan - and thus, downstream evolutionary history could have been very different. In Gould's eyes, the (first ${ }^{10}$ ) reinterpretation of the Burgess Shale (along with his assumption of fitness-insensitive sampling) constituted evidence for the biological evitability of forms. Thus, if the Burgess Shale supports evolutionary contingency, then it is in virtue of its support for the biological evitability of forms.

Even beyond evolutionary biology, biological evitability is of concern. For instance, the truth of biological evitability appears to be particularly pressing for Conway Morris's theology $(1998,2003)$, for it seems to threaten the theistic significance of humans: after all, if evolutionary forms were evitable, then humans might not have evolved. How can theism be reconciled with the arbitrariness of humans as products of shaky historical accidents? Clearly, there is a lot riding - theologically speaking - on the biological evitability of forms. It is no surprise, then, that Conway Morris (2003) takes himself to be arguing for the biological inevitability of forms, as suggested by title of his book: "Inevitable Humans in a Lonely Universe" (emphasis added). But if a sense of biological evitability is not subsumed under evolutionary contingency, then has Conway Morris been arguing against a strawman? That is - if evolutionary contingency does not specify the biological evitability of human evolution (but merely pointed to the etiological dynamics of the causal chains that led up to human evolution), then humans can be deemed 'evolutionarily contingent' yet inevitable all the same.

To strengthen the case even further from a different perspective: there was a commonality to both versions of Gould's replay experiment. That is - both versions of Gould's metaphor, regardless of whether there was an alteration in initial conditions, stipulated a difference in outcomes. This would explain why Gould seemed to nonchalantly slip between different versions of the tape of life metaphor: perhaps, it did not matter so much to Gould as to whether initial conditions are altered insofar as the outcome would (probably) turn out differently.

Thus far, I have presented reasons for the importance of biological evitability in an account of evolutionary contingency: past discussions of contingency have implied biological evitability

\footnotetext{
${ }^{9}$ Curiously, nowhere in Wonderful Life (1989) does Gould offer any explicit justification as to why the sampling of Cambrian species was fitness-insensitive.

${ }^{10}$ Owing to a transition from evolutionary systematics to cladistics, an adoption of quantitative morphological methods, and new discoveries in China and Greenland, it has been alleged that Wonderful Life was very much a product of its time in that the latest paleontological data paints a picture of the Cambrian as possessing less disparity than what Gould originally envisioned.
} 
(including situating itself well within the Gouldian narrative ${ }^{11}$ ) and it allows for the contingency to be relevant with related debates. There is a third reason: after developing the idea of trajectory propensity in a dynamical systems framework, I shall argue that some biological processes, which account for the probability of initial conditions but not the etiological structures (e.g. causal dependence, sensitivity to initial conditions, etc.), are excluded unless evolutionary contingency satisfies the feature of biological evitability. For now, I return to Beatty's (2006) causal dependence sense and argue that it fails to entail biological evitability.

Despite its emphasising the importance of history, recall that the causal dependence sense, which Beatty (2006) interprets from Gould's replay experiment, was also supposed to portray a difference in outcome: "[a]ny replay, altered by an apparently insignificant jot or tittle at the outset, would have yielded an ... outcome of entirely different form" (Gould, 1989; emphasis added). But, drawing on a meticulous distinction between questions of causal sufficiency, causal necessity, and metaphysical necessity noted by Ben-Menahem (2009) in a parallel discussion in the philosophy of history, it is easy to see that Beatty's sense of causal dependence, per se, is insufficient to entail the biological evitability of outcomes. Even if an antecedent was causally necessary for an outcome, it does not say anything about the modal status of the antecedent - that is, the degree to which it is necessary or contingent. Nor does it follow that the antecedent was causally sufficient for the outcome (to think so would be to commit the 'converse fallacy').

The point is that just because an antecedent $A$ is causally necessary for $O$, it does not follow that the antecedent $\mathrm{A}$ necessarily gives rise to $\mathrm{O}$, nor that the antecedent will always occur, nor that the antecedent never occurs. Following standard philosophical tradition, the 'causal sufficiency of the antecedent', the 'causal necessity of the antecedent' and the 'metaphysical necessity of the antecedents' are all distinct notions. Accordingly, even if the causal dependence sense was true for an evolutionary form such that a specific antecedent was causally necessary for that form's occurrence (e.g. the evolution of wings requires body plan X), the antecedent may be such that it always occurs. In this way, the causal dependence sense, per se, is insufficient to guarantee the biological evitability of outcomes. À la Ben-Menahem, we must not confuse causation with historical inevitability! The mistake is analogous to the common conflation of various properties about causality with fatalism in the metaphysical determinism literature.

However, there is a way in which 'causal dependence' can lead to evitable outcomes, and it is to appeal to the chancy nature of a special class of historical events ${ }^{12}$. This is, in essence, the Gouldian strategy found in Wonderful Life. That is - contingency as causal dependence can be complemented with appeals to a special kind of historical events (Gould calls them 'historical constraints'), which include some (but not all) sources of contingency. These historical events are supposedly quirky and metaphysically contingent, whereby plausible candidates include drift outcomes, mass extinctions, recombination outcomes, and mutational outcomes. So, even if an evolutionary outcome was causally dependent on an antecedent (in Beatty's 2006 sense), insofar as the antecedent is chancy in its occurrence, there is some room for evitability. This strategy is akin to combining Beatty's unpredictability sense (though, specifically, for the antecedent) and the causal dependence sense

\footnotetext{
${ }^{11}$ This is, of course, only an exegetical reason for the importance of the feature of biological evitability.

12 Interestingly, later in 2011, Beatty in a co-authored paper with Carrera comments on the inadequacy of causal dependence, alone, to capture the chanciness of evolutionary outcomes and suggests that causal dependence ought to be combined with a sense of 'unpredictability', but not the sense hitherto considered whereby the outcomes are 'unpredictable'. Rather, Beatty suggests that it ought to be combined with a sense whereby the antecedents are 'unpredictable'.
} 
(Beatty, 2006). However, it remains that causal dependence, by itself, is simply uninformative about the evitability of biological forms.

Put another way, the causal dependence sense, by itself, is somewhat trivial, for all it says is that outcomes are 'causally dependent' on the events that preceded them, such that: if the past had been different, then the future would also be different. Whilst it is true that some changes in initial conditions will produce some changes in outcome, it is not clear that there would be such changes in initial conditions at all: even if the future does depend on the past in the specific way that the causal dependence sense requires, on what basis would there be a chance in initial conditions? The moral of the story has been that there is no such basis: causal dependence does not necessarily entail evitable outcomes. Importantly, whether it is probable for the initial conditions to change at all is concerned with relevant facts about those initial conditions and can be formalised by the concept of trajectory propensity under a dynamical systems analysis.

\section{Two Forms of Modalities: Trajectory Propensity and Counterfactual Robustness}

Let us begin with a quotidian example involving train tracks to introduce the idea of trajectory propensity, and then consider a more sophisticated counterpart in dynamical systems, which affords additional complexity for modal analysis. At London St. Pancras station, there are a series of train tracks that lead to various destinations: Paris, Brussels, Amsterdam, and so on. Each of these train tracks may exhibit certain properties. For instance, quite plausibly due to facts of geography, the track(s) to Paris may necessarily pass through Lille, and the track(s) to Amsterdam may necessarily pass through Antwerp. There is 'causal dependence', so to speak. To reach Paris on a train, one must have passed through Lille.

Moreover, a set of tracks may also exhibit properties (population-level property of tracks). For instance, even though all the tracks at St. Pancras are closely related in space to each other (they find themselves side by side inside the station, after all), they may reach destinations that are distantly related. In fact, we would take this to be an ordinary feature of any good train station lest it be of little use for international travel. But, because of this feature, a passenger who takes a few steps towards the wrong platform (and hence, boards the wrong train) could end up at a destination markedly distant from their intended destination. There is sensitivity to initial conditions, so to speak.

However, it is easy to tell from this example that passengers do not so easily head towards the wrong platform, board the wrong train, or arrive at the wrong destination. Barring far-fetched Kafkaesque stories, passengers are ordinarily able to make their way to the right platform, trains function normally, and the tracks are reliable. After all, passengers listen to announcements and check reliable station signage, and regular maintenance is performed on the trains and the tracks. That is - there are good reasons why a passenger will tend to end up at their intended destination. Biology, I submit, is not too different. There may be good biological reasons for why certain evolutionary trajectories are highly probable regardless of whether those (set of) trajectories structurally exhibit causal dependence, sensitivity to initial conditions, or path dependence.

\subsection{The Dynamical Systems Analysis}

Progressing from the toy example, imagine an evolutionarily dynamical system whereby there are various trajectories, depicted by lines, leading continuously from point to point in phase space (see Figure 2). The dynamical system contains as many dimensions as needed to capture all the relevant aspects of the system, and, as such, phase points in the dynamical system exhaustively describe states of the system. What it means for a certain history to have obtained is just to be found in a 
specific phase point of the system. Trajectories are a sequence of $x_{0}, x_{1}, x_{2}, \ldots, x_{n}$, where $x$ are phase points and $x_{0}$ is the initiating phase point (or, colloquially, the 'initial conditions') of any particular trajectory. The shape and direction of trajectories (i.e. the topography) are determined by the relevant nomological laws pertinent to the system such that if natural selection, for example, had been one way rather than another, then trajectories may be one shape rather than another.

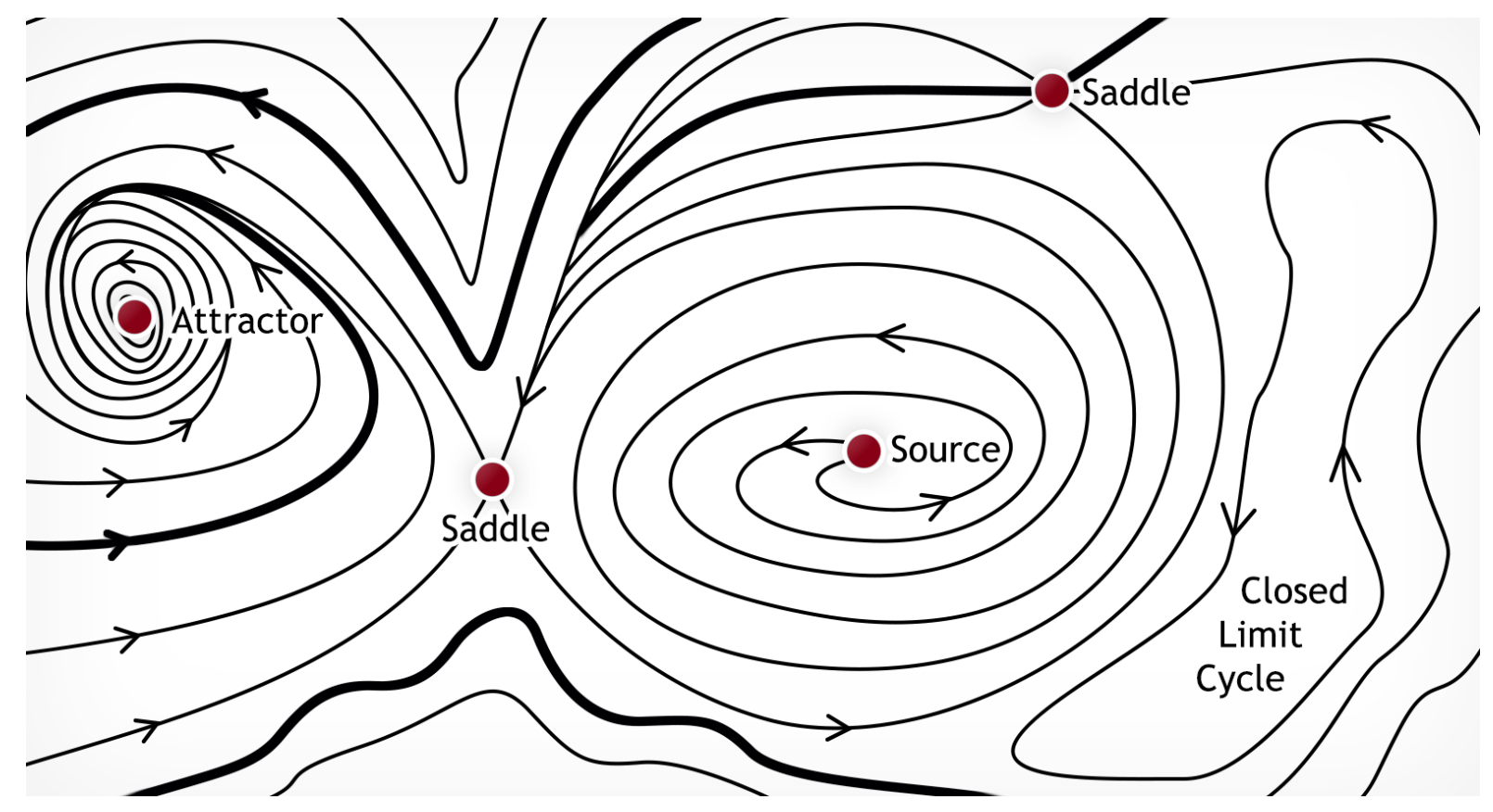

Figure 2. Two-dimensional Cross-Section of an Evolutionarily Dynamical System ${ }^{13}$

There is, indeed, some visual resemblance between evolutionary trajectories and train tracks from the bird's eye view. But just as the probability of a passenger travelling on a specific track depends on the accuracy of station signage, reliability of train tracks, and much else, trajectories in an evolutionarily dynamical system are more or less probable depending on the features of the phase points of the trajectory. I will come to elaborate on these features shortly.

But, for now, the dynamical systems framework is useful to illustrate two distinct forms of modalities that have been implicit in discussions of evolutionary contingency. On one hand, there is the matter of counterfactual robustness, which has been the primary object of attention in past discussions of evolutionary contingency. Roughly, some phase point (or trajectory ${ }^{14}$ ) is counterfactually robust if it obtains despite a variation in system conditions. That is - the extent to which an event is counterfactually robust is determined by its persistence in light of perturbations to (earlier) system conditions. On the other hand, there is something distinct; there is the probability of an evolutionary trajectory obtaining in an evolutionary system, which one may call trajectory propensity $^{15}$.

Trajectory Propensity is the probability of a trajectory obtaining within a system. It is equal to the product of the probability of all continuous phase points of the trajectory such that a

\footnotetext{
${ }^{13}$ This particular system is continuous and non-ergodic.

${ }^{14}$ Trajectories can also be counterfactually robust when multiple trajectories converge into one (see Figure 2).

15 I do not mean to imply that probabilities must be interpreted as propensities.
} 
decrease of the probability of any phase point of the trajectory is accompanied by a decrease in the trajectory propensity (ceteris paribus) ${ }^{16}$.

Relating this to the train example, it might be said that there is some counterfactual robustness in the ways that a passenger can make it to their intended destination. That is - if the original route is no longer functional, then, perhaps, another route can be taken to reach a passenger's intended destination. It is this 'multiple paths to the same destination' that accounts for counterfactual robustness. However, it is the reliability of station signage, trains, and train tracks that pertain to trajectory propensity. Thus, it may be the case that, despite there being many paths to Paris, an alternative route need not ever be taken because the initial route was highly reliable.

Likewise, there are two types of modalities at play for evolutionary systems: firstly, there is the matter of (i) trajectory propensity (i.e. how easily can system progressions remain on a trajectory, or switch to another) and, then, the (ii) counterfactual robustness of phase points or trajectories (i.e. the extent to which perturbations in system conditions fail to render a difference in the occurrence of phase points/trajectories). The second corresponds to the form of modality concerned when initial conditions are said to have been altered. However, when replaying the tape (whether it is the tape of St. Pancras or the tape of life), both forms of modality matter.

In our dynamical systems analysis, counterfactual robustness is evident when a particular phase point can be reached by a number of different trajectories. Attractors, saddles, and other equilibrium points are paradigmatic examples of phase points that have high counterfactual robustness (see Figure 2 ) $^{17}$. For example, if a different set of facts obtain such that phase point $y_{1}$ instead of phase point $x_{1}$ obtains, but the same outcome occurs in either scenario, then the outcome is counterfactually robust. The extent to which it is counterfactually robust depends on the number of alternative trajectories by which it can be reached.

In the past, the second type of modality (i.e. counterfactual robustness) has been famously alluded to in explicitly dynamical terms. Waddington $(1939,1940,1957)$ proposed the idea of the epigenetic landscape (a developmental instance of our general evolutionary system) and spoke of 'canalization'. According to Waddington, there is canalization when the developmental nomologies of the system stipulate that there are different trajectories on the epigenetic landscape that, quite literally, 'funnel' into a specific point in phase space. Genetic canalization, then, is just a specific instance of how counterfactual robustness can be exhibited in such a dynamical systems framework.

The distinction between the two forms of modalities highlights the fact that even if there is little counterfactual robustness, an evolutionary trajectory, on account of its high probability, can be quite certain to occur. In fact, there are biological scenarios where there are evolutionary trajectories that are highly probable to obtain regardless of whatever complex etiological structure they may be embedded in.

To see this, recall that trajectory propensity (as per the definition above) is dependent on the probabilities of the individual phase points that make up the trajectory. In turn, the probabilities of

\footnotetext{
${ }^{16}$ One way of interpreting probabilities in a dynamical systems analysis is to imagine a Gibbsian ensemble (1902), which refers to an infinite array of identical copies of the dynamical system, each of which are in a particular, unspecified system state. The probability of a phase point would, then, be akin to the probability of randomly picking out a system from this ensemble that is at the system state of being at that phase point. ${ }^{17}$ Sterelny's (2005) concept of conditional inevitability would just be a case whereby all trajectories that pass through a condition will lead to a certain attractor. In other words, if the condition is satisfied, then that outcome will be reached.
} 
individual phase points are dependent on the features of those phase points ${ }^{18}$. In other words, depending on the conditions that a phase point describes, it may be more or less probable than other phase points. So, for example, recent studies suggest that our four RNA nucleobases (i.e. Adenine, Uracil, Guanine, Cytosine) were likely to come about on account of formamide's chemical tendency to produce specifically those four RNA bases (e.g. Ferus et al., 2015, 2017; Saladino et al., 2001). At the same time, formamide was present in sizable amounts during Earth's early history (Ibid.) Accordingly, in our dynamical systems analysis, phase points that contain in their descriptions our four RNA bases may be more probable than phase points that do not (ceteris paribus). If this is true, then trajectories that contain phase points with our four RNA bases have greater propensity than trajectories that contain phase points that do not (ceteris paribus). In general, an evolutionary trajectory composed of a sequence of highly probable phase points can be said to have high trajectory propensity - which, purely as a form of visual demonstration, can be illustrated by thicker lines in Figure 2.

Consider a different biological example involving natural selection: take the phase point whose description of the system includes facts pertaining to the adaptive landscape (sensu Wright) in which a species is located. Some adaptive landscapes are more probable than others due to facts of the environment and the relevant nomological laws. This is, partly, owing to the fact that environments are often composed of other species (Lewontin, 1983) whose existence and behaviours may be quirky themselves. For example, in cases of Batesian or Mullerian mimicry whereby there is an evolution of apparently arbitrary colour patterns in order to mimic other species in the environment, whether a particular colour pattern is, indeed, an adaptive peak is dependent on the existence of other species and whatever patterns they may have. But, perhaps, certain species are unlikely to be found in that environment for ecological reasons, or that, due to certain facts about protein synthesis for pigmentation, some variations of colour patterns are less likely to come about. If this is so, then the specific colour patterns are not so arbitrary after all. The point is that, depending on features of a state of the system, the adaptive landscapes and their adaptive peaks specified by phase points may vary in their probability. In this case, it is not even certain where natural selection is aiming towards - the determination of which is amenable to probability.

Recall that, earlier, I argued that causal dependence can come apart from biological evitability. This is now especially clear under the dynamical systems analysis. Much like the train example, certain etiological dynamics may be exhibited in an evolutionarily dynamical system ${ }^{19}$. Consider Figure 3 , whereby causal dependence for $\mathrm{O}_{1}$ is exhibited since any trajectory that reaches $\mathrm{O}_{1}$ necessarily passes through $\mathrm{A}_{1}$ (but not vice versa).

\footnotetext{
${ }^{18}$ Features of phase points also include time indices. Hence, evolutionary trajectories are ordinarily also temporal progressions since if $\mathrm{x}_{0}$ has time $\mathrm{t}$, then $\mathrm{x}_{1}$ will have ordinarily have time $t+1$. In principle, some trajectories may also be reversible - but this would, of course, be contrary to Dollo's law.

${ }^{19}$ Etiological dynamics may be true globally within a system or only within certain locales. This distinction does not matter for our purposes here.
} 


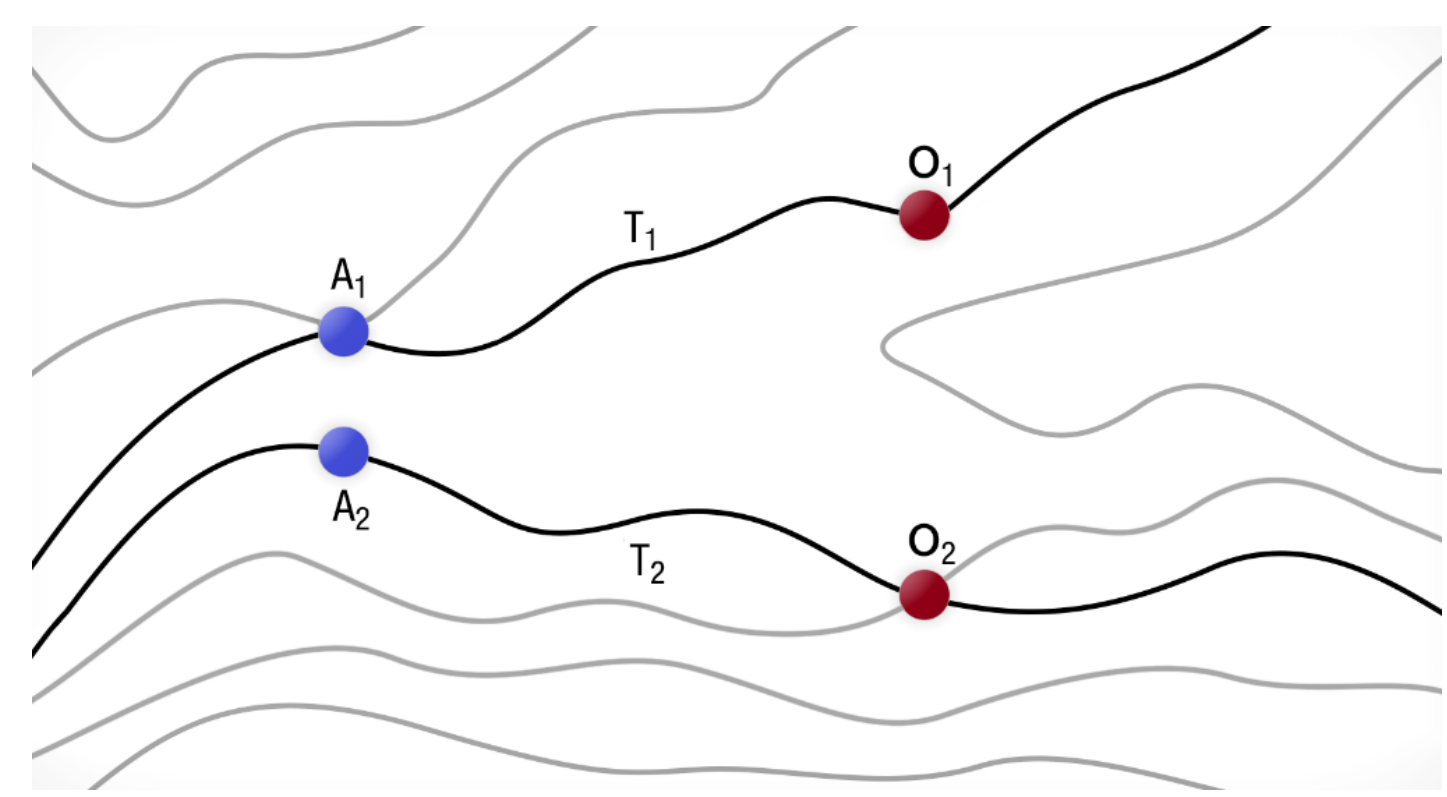

Figure 3. Micro Cross-Section of a Non-Ergodic Dynamical System (depicting 'Causal Dependence' for $\mathrm{O}_{1}$ )

It is easy to see that even if causal dependence is true for $O_{1}$, if trajectory $T_{1}$ has high trajectory propensity, then $\mathrm{O}_{1}$ is highly probable to obtain. Perhaps, features of phase points found on $\mathrm{T}_{1}$ render that it has high propensity vis-à-vis $\mathrm{T}_{2}$, and thus replays of the system will, probably, result in the occurrence of $\mathrm{O}_{1}$ rather than $\mathrm{O}_{2}$. It is a neglect of the idea of trajectory propensity that has led to a failure to distinguish Beatty's (2006) causal dependence from the nomic certainty of initial conditions in a modal analysis of biological forms. The possibility of high trajectory propensity presents reason to doubt that etiological structures, on their own, like the causal dependence sense can lead to evitable outcomes. And, if trajectories with high propensity turned out to be pervasive in the biological world, then accounts of evolutionary contingency that neglect trajectories with high propensity would have too wide a scope; that is, these accounts would have recognised more evolutionary contingency than there actually is in the biological world.

However, the etiological structure of a system remains a determinant component for the evitability of biological forms. This is because whether an outcome is counterfactually robust depends on the etiological structures of the surrounding region within the dynamical system. For example, if a phase point is absolutely insensitive to initial conditions, in that all trajectories reach it, then there is maximal counterfactual robustness. Thus, in addition to the propensity of trajectories, the etiological structures of the (set of) trajectories are also relevant.

Given these considerations, the biological evitability of an outcome is some function of both the modal components of (a) trajectory propensity and (b) counterfactual robustness, which is in turn determined by the etiological dynamics of the (set of) trajectories that lead up to the outcome. The latter has been the primary focus of previous discussions of evolutionary contingency. However, to exclude either modal component would be to commit a conceptual oversight concerning what it is to be biologically evitable. The causal dependence sense, per se, was guilty of neglecting the former component. And so, an appreciation of trajectory propensity affords a more complete analysis of the modality of biological forms than previously recognised.

This distinction can also help make sense of existing views. Conway Morris's (2003) chief claim is that humans become inevitable once life on Earth has begun. However, Conway Morris (2003) remains indifferent to whether life on Earth is bound to occur; in other words, it has been very much left as 
an open question. In fact, the (apparent) uniqueness of intelligent life might suggest that such initial conditions are nomically improbable. Nonetheless, Conway Morris (2003) can be interpreted as arguing for the counterfactual robustness of humans whilst remaining silent on the issue of trajectory propensities.

Moreover, this distinction between modalities would help explain Gould's insistence on discounting stochastic processes, such as genetic drift, as sources of contingency whilst affirming the importance of historical particulars (c.f. Beatty, 2006). Most varieties of genetic drift would account for only trajectory propensity, whilst the question of which historical particular obtains would account for only counterfactual robustness. Perhaps, Gould had in mind only counterfactual robustness when thinking about contingency.

Before I analyse some other senses of contingency in light of the notion of trajectory propensity, there is a worry that the recognition of trajectory propensity as distinct from counterfactual robustness has been merely a matter of perspective. That is - facts that were relevant to the propensity of trajectories (e.g. features captured by the trajectories' phase points) can be simply recast as facts that are relevant to an outcome's counterfactual robustness. If this is true, then the biological evitability of an outcome can be fully accounted for by the conditions of the etiological structures without the need for a superfluous notion like trajectory propensity. This is false, and I submit that there is a real conceptual distinction between trajectory propensity and counterfactual robustness for a given model.

Sober's (1988) bowl analogy is illustrative of such a distinction. A bowl with a single equilibrium state (i.e. with only one well at the bottom) is said to have an outcome that is counterfactually robust. This is because wherever a marble is released at the top of the bowl, the marble will rest in the same place at the bottom of the bowl (i.e. there is an equilibrium point). As such, what explains this counterfactual robustness is (inter alia): the force of gravity, the shape of the bowl, and its having only one well. But these facts are distinct from the facts that determine where the marble is released (e.g. facts determining where the agent's hand goes). It was true that given that the outcome is insensitive to conditions, where the marble is released does not affect which outcome is realised. But, now, suppose that the bowl has two wells such that there is some sensitivity to initial conditions: let us say that if the marble is released on the left side, it ends up in the left well, and if its released on the right side, it ends up on the right well. Further suppose that our agent is a righthanded individual and will, quite consistently, release the marble on the right side. Under these circumstances, out of all the trials that one can conduct, the marble will almost certainly rest in the well that is on the right. But, the point, however, is that, within this model, the facts that explains the counterfactual robustness (e.g. gravity, shape of bowl, number of wells, etc.) are clearly distinct from the fact(s) that determine where the marble is released, namely, that the agent is righthanded. So, trajectory propensity is distinct from counterfactual robustness for any given mode ${ }^{20}$.

Also hinging on this distinction of modality is the reason why certain 'sources of contingency' are, problematically, theoretically excluded unless evolutionary contingency satisfies the feature of biological evitability. The examples previously presented (viz. fluctuating adaptive landscapes and RNA nucleobases) account for only trajectory propensity and not for any of the etiological dynamics of the system at hand. That is - they do not explain why certain (sets of) trajectories behave the way they do, but only that certain trajectories are more or less probable. For example, the reason why

\footnotetext{
${ }^{20}$ Even if one were to incorporate 'handedness' into the model, there would still be the probability of an agent being right-handed (demographic data indicates that it will be quite high). The point is that no matter what is to constitute the initial conditions, there will be some nomic probability for those initial conditions.
} 
the nucleobases ' $A, U, C, G$ ' are more probable than alternative nucleobases to constitute RNA does not explain the ostensible fact that 'had the constitution of RNA been just slightly different, then life would have been markedly different'.

As such, if evolutionary contingency did not entail evitability but merely asserted certain etiological structures, then the sorts of biological phenomena that account only for trajectory propensity in their determination of evitability are excluded. In other words, if sensitivity to initial conditions is all that there is to evolutionary contingency, then the nomic regularity of RNA bases, fluctuating adaptive landscapes, and so on. are irrelevant since they do not explain sensitivity to initial conditions. Accordingly, this serves as an additional reason for why biological evitability is a desideratum of evolutionary contingency: some sources of contingency ordinarily taken to account for contingency are theoretically excluded unless evolutionary contingency satisfies the feature of biological evitability.

The key point of this section, thus far, has been that biological evitability is dependent on both trajectory propensity and counterfactual robustness. And, I have shown that Beatty's (2006) causal dependence neglected trajectory propensity. However, contra their respective advocates, many of the other major accounts of contingency also fail to entail evitable outcomes due to a neglect of trajectory propensity. I shall now enlist a series of demonstrations to show how these senses of evolutionary contingency also fail the biological evitability desideratum.

\subsection{Trajectory Propensity vis-à-vis Other Accounts of Evolutionary Contingency}

The interpretation of evolutionary contingency that has garnered the greatest following is contingency as 'sensitivity to initial conditions' (e.g. Ben-Menahem 1997, 2009; Sterelny 2005; Powell, 2012; Powell \& Mariscal, 2015). Sensitivity to initial conditions is similar to causal dependence in that they both attribute causal power to the antecedent events in determining outcomes (and hence, emphasise the importance of history), but they are not the same. For an outcome that is 'causally dependent' (Beatty, 2006), there is a causally necessary antecedent, and, furthermore, if that antecedent were to be different, then there would be a different outcome. Sensitivity to initial conditions is a stronger notion that also admits of gradations.

There is sensitivity to initial conditions, if minor changes to initial conditions are sufficient to elicit drastic changes in outcome. In contrast, an outcome that is wholly insensitive to initial conditions entails that the event is a necessary occurrence in that system: regardless of the initial conditions that hold, the event will occur all the same.

Often touted as its virtue, sensitivity to initial conditions does not prima facie have any tensions with determinism since it does not assert that the same initial conditions yield different outcomes. Rather, markedly different outcomes result from similar initial conditions whereby these initial conditions may or may not have uniquely determined their outcomes. Sensitivity to initial conditions is neutral with respect to determinism. 


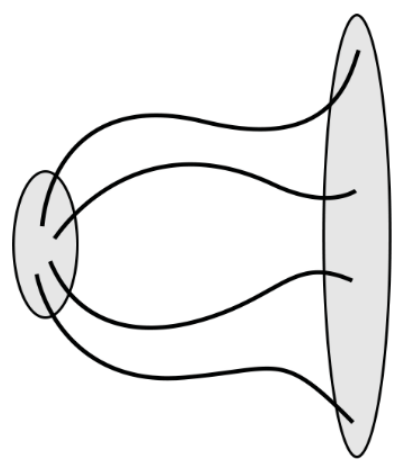

Figure 4. Sensitivity to Initial Conditions

However, sensitivity to initial conditions is also subject to the same shortcoming as the causal dependence sense in that it, on its own, fails to entail a difference in outcomes. This is because there may be initial conditions that are nomically probable, such that they are conducive to high trajectory propensity (of trajectories in which they are a part). The fact that an outcome is sensitive to initial conditions is simply uninformative with regards to whether or not certain initial conditions are nomically probable. That is - even though an evolutionary outcome may be highly sensitive to differences in initial conditions, what reason is there to suppose that a different trajectory would have been realised? A high sensitivity to initial conditions is wholly compatible with an inevitable outcome if the trajectory that leads to that outcome has maximal or minimal propensity. For example, suppose that the evolution of an adaptive complex depended on the body of genetic material contained in a particular genome, $G_{1}$. But, further suppose that $G_{1}$ differs minutely to another genome, $G_{2}$, by only one base pair. As such, the outcome - namely, the evolution of the adaptive complex - is highly sensitive to initial conditions. However, despite this sensitivity to initial conditions, the adaptive complex may not be likely to come about. Perhaps, high-fidelity errorchecking mechanisms make it unlikely that $\mathrm{G}_{2}$ is ever prevalent enough in the population for the adaptive complex to evolve. Clearly, sensitivity to initial conditions is not enough for the evitability of forms.

I, now, consider path dependence, and make a distinction between Type I path dependence as it is commonly used in the economics literature (e.g. Arthur, 1989; Liebowitz and Margolis, 1995; David, 2001) and a more sophisticated account, Type II path dependence, developed by Desjardins (2011a, $2015,2016)$. The former is used in economics and the social sciences to demonstrate that (arbitrary) choices of earlier paths can lead to 'lock-in' effects and market failures. However, this type of path dependence, despite its emphasising the effects of the past on the future, can nonetheless result in inevitable outcomes. This is because, as I shall explain, Type I path dependence turns solely on the existence of alternative paths from an initial state, the probabilities of which change as a result of previous realisations. Desjardins' path dependence, however, entails the evitability of outcomes since it explicitly stipulates that there are multiple possible outcomes.

Type I path dependence asserts that travel on certain paths precludes or reduces the probability of travel on downstream paths and entails that there must be multiple alternative paths from an initiating point. In other words, an outcome is Type I path dependent if and only if it is at the end of a causal chain that changes as a function of prior events realised. An example definition:

Path Dependence (Type I): A path dependent stochastic process is one whose ... distribution evolves as a consequence (function of) the process's own history. (David, 2001; emphasis added) 
However, this definition does not require that there be multiple outcomes: insofar as there are alternative paths from the initiating point, and the probability of downstream paths changes as a consequence of previous path realised, then there is Type I path dependence ${ }^{21}$. The below branching diagram illustrates an instance of Type I path dependence, as is consistent with the above definition. But notice that there is only one outcome; $\mathrm{O}_{1}$ is inevitable in that it will always occur no matter the paths that are realised to reach it. However, this system may be said to be transiently path dependent; that is, the realisation of $B_{1}$ or $B_{2}$ does affect the probabilities of events at intermediary stage $C$. Nonetheless, although it may serve its purpose in the economic or social science literature in emphasising the importance of history, since Type I path dependence can fail to entail the evitability of its outcomes, it fails as an account of evolutionary contingency.

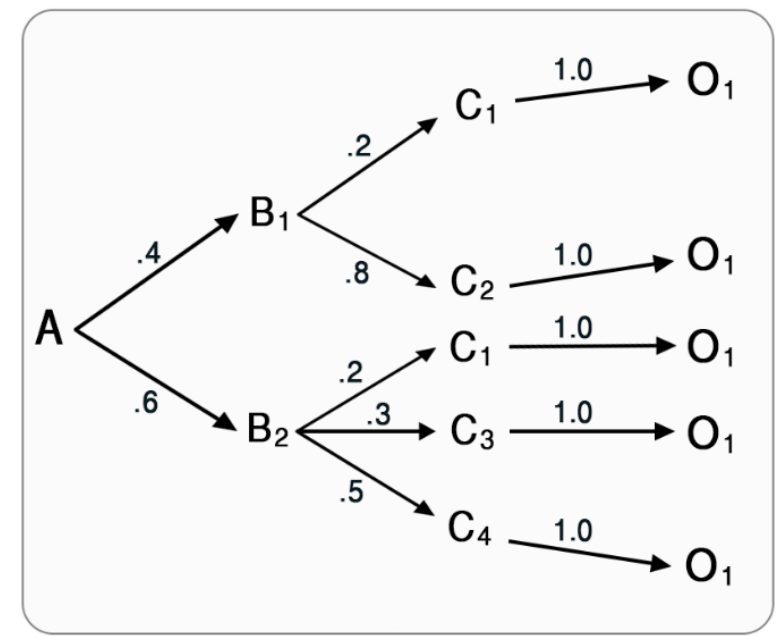

Figure 5. Type I Path Dependence Branching Diagram

There is, however, an account of path dependence that entails the evitability of forms since it requires there to be multiple outcomes with non-trivial objective probabilities of occurrence ${ }^{22}$. Desjardins (2011a) proposes this account, which attributes non-trivial (other than 1 or 0 ) probabilities to outcomes, and employs a branching diagram to illustrate:

\footnotetext{
${ }^{21}$ It is unclear whether David (2001) intended to necessitate the multiplicity of possible outcomes or whether he meant to leave it an open question. In the same paper, he offers a 'negative definition' of path dependence by specifying its non-ergodicity which entails that there is not an invariant probability distribution that is continuous across all possible instances. Consistent with non-ergodic systems, however, is that there is only one possible outcome at the last instance. So, even his negative definition of path dependence is Type I. 22 There may be a background issue here in that disagreement exists concerning whether non-trivial objective chances are congruent with determinism. We have come full circle to the indeterminism issue.
} 


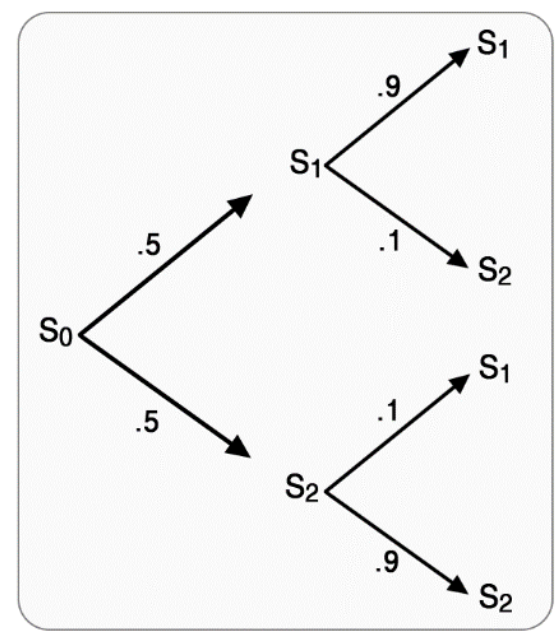

Figure 6. Branching Tree of Type II Path Dependence (Redrawn from Desjardins, 2011a) The numbers next to the arrows specify the probability of that path.

From the branching diagram, one can see that the probability of the outcome, either $S_{1}$ or $S_{2}$, changes with the path taken by the process. Incidentally, in this example, the probabilities of the outcome $S_{1}$ and $S_{2}$ are the same at the outset (i.e. 0.5), but their respective probabilities change as soon as the first splitting event. However, importantly for our purposes here, there is more than one possible outcome (namely, $\mathrm{S}_{1}$ and $\mathrm{S}_{2}$ ) since there are non-trivial (other than one or zero) probabilities specified for these two outcomes: i.e. 0.5 and 0.5 (they need not have different probabilities). From the branching diagram, both $\mathrm{S}_{1}$ and $\mathrm{S}_{2}$ are evitable outcomes.

Powell (2012) initially proposes a 'sensitivity to initial conditions' account that fails to entail evitability for the reasons mentioned previously. However, Powell appears to recognise this shortcoming in a later co-authored paper (Powell and Mariscal, 2015), and applies the quick and easy fix of stipulating that the initial conditions of an evolutionarily contingent outcome ought to be of low objective probability. In this way, the antecedents of an evolutionarily contingent outcome are ex hypothesi "unlikely to be replicated across the vast majority of alternative evolutionary histories" (Ibid). This amended 'radical contingency' account (Ibid.) effectively couples low trajectory propensity with a high sensitivity to initial conditions, such that the outcomes are, now, evitable.

\section{Contingency as Non-Trivial Objective Probability}

Thus far, I have argued that biological evitability is an important desideratum for an account of evolutionary contingency and that some accounts of contingency have failed in this respect. In the second half of this paper, I shall characterise an understanding of evolutionary contingency as nontrivial objective probability. This new account shall be compared and contrasted with previous proposals and, then, evaluated in light of some desiderata. It shall be seen that the new account satisfies all three desiderata. However, it is not the only account that is able to do so: Desjardins' path dependence $(2011 a, 2015,2016)$ account fares equally just as well. Although there are some differences between the path dependence account and the non-trivial objective probability account in terms of how they emphasise the importance of history, there is no obvious way to adjudicate between them for an account of evolutionary contingency. Perhaps, there is to be a sense of plurality for evolutionary contingency; for now, I leave it an open question.

In what is to follow, I identify two additional desiderata, both of which are demanded by certain normative and scientific considerations: (i) being able to vary in degree and (ii) subsuming historicities. It should be said that the three desiderata (including biological evitability) considered in 
this paper are not necessarily exhaustive; that is - there may be other desiderata that are not presently entertained ${ }^{23}$. But, as we shall see, the various accounts of evolutionary contingency can already fare quite differently with respect to the three desiderata (this is summed up later in Table 1).

\section{Evolutionary Contingency as Non-Trivial Objective Probability: An outcome is evolutionarily contingent if and only if it has a non-trivial (not 1 or 0 ) objective total probability within a specified modal range}

There are a few crucial ideas to unpack in this definition. Firstly, evolutionary contingency would be concerned with the objective probability, as opposed to the subjective probability, of an outcome's evolution. In other words, evolutionary contingency would not be an epistemic notion that is concerned with what evolutionary biologists, given their knowledge, can predict about how probable (or often) an outcome occurs. Rather, the definition is such that evolutionary contingency is about the fact of the matter regarding the probability that an outcome evolves, which is in turn governed by the relevant evolutionary dynamics, natural facts, and historical particulars.

Secondly, evolutionary contingency would be about the unconditional probability (or, what I call, total probability, for reasons to become apparent) of an outcome. This is in contrast to conditional probabilities, which are inherent to Desjardins' path dependence account. This distinction is important because conditional probabilities of an outcome do not change when certain modal facts change (e.g. the addition of alternative trajectories), whereas total probabilities do change.

Thirdly, by virtue of asserting non-trivial (not 1 or 0 ) probabilities, this conception already satisfies the desideratum of biological evitability, as it precludes necessity in either an evolutionary outcome obtaining or not obtaining. In other words, if an outcome does not have 1 or 0 objective probability, then it is genuinely uncertain whether it will evolve.

Lastly, with the help of the modal range (Wong, 2019), this account offers an explicit way to specify the extent (geographically, nomologically, counterfactually, etcetera) to which contingency allegedly extends. In other words, the modal range defines the reference class of the probability statements inherent in contingency claims and, thus, outlines their satisfaction conditions. Under a frequentist interpretation, the probability would be just how many times the form evolves out of the total number of evolutionary scenarios in the modal range ${ }^{24}$.

In line with evolutionary contingency being a naturalistic thesis about how evolution behaves (rather than some mysterious metaphysical thesis), an outcome's objective probability of evolution is dependent on the evolutionary dynamics that govern the outcome's evolution within a specific modal range. As per section 4, these dynamics are, in turn, determined by various natural facts and histories pertinent to the evolution of that outcome within a specific modal range. The contention within the contingency debate, then, is whether the evolutionary dynamics are, indeed, sufficient to entail with high probability that certain outcomes evolve. The hard adaptationists would claim precisely just that. For them, the evolutionary dynamics comprise of a 'strong' sense of natural selection-cum-variation that is able to reach the highest of adaptive peaks. As a result, certain outcomes (namely, optimal traits) have a high objective probability of evolution (within the modal

\footnotetext{
${ }^{23}$ One need not exhaustively consider the full complement of desiderata (which I imagine is a matter of contention in and of itself) in order to put forth the new account for consideration.

${ }^{24}$ Even under a (single or long-term) propensity interpretation of probability, frequencies of outcomes would be indicative of the likelihoods (technical sense) of various propensity probabilistic theses. For the purposes of this paper, a distinction between various interpretations of probability need not be made.
} 
range); the evolutionary dynamics of a system will reliably guarantee their occurrence. Equally, however, the evolutionary dynamics are a function of the various histories that impinge. After all, phase points describe not just natural facts but historical facts. Therein lies the empirical disagreement between the contingentists and non-contingentists.

Relatedly, notice that, under the proposed account, the probability of an evolutionary outcome also depends on the past. More specifically, in accordance with the chain rule, an outcome's probability is also determined by the product of the probabilities of preceding phase points and/or transitions (this is congruent with dynamical trajectory propensity). To see this, consider the following two-step causal pathway:

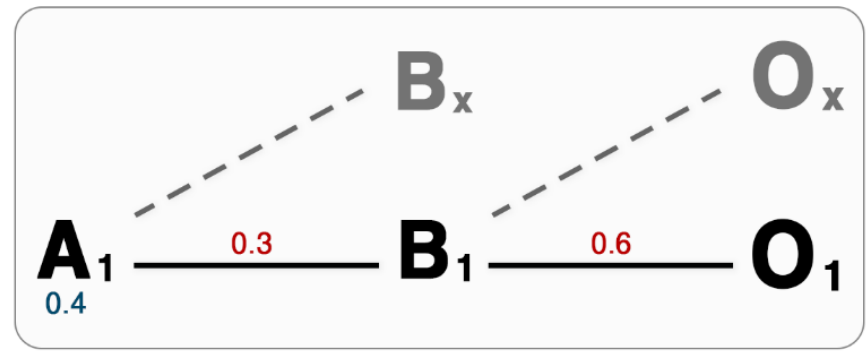

Figure 7. Causal Pathway of $\mathrm{O}_{1}$

This particular example is two-stepped because the final outcome, $\mathrm{O}_{1}$, is preceded by two events: $A_{1}$ and $B_{1}{ }^{25}$. The dotted grey lines indicate that $A_{1}$ could lead to some other event(s), $B_{(x)}$, and, $B_{1}$, itself, can lead to $O$ events other than $O_{1}$. That $A_{1}$ and $B_{1}$ can lead to other events, which is just to say that there are alternative possibilities from $A_{1}$ and $B_{1}$, can also be inferred by $B_{1}$ and $O_{1}$ possessing an objective probability of less than 1 . The blue values represent unconditional probabilities of an event whilst the red values represent transition probabilities (or conditional probabilities of one event from another).

Consider the possible transition from $\mathrm{B}_{1}$ to $\mathrm{O}_{1}$, where the transition probability is 0.6 . As before, this transition and its probability are determined by the relevant nomologies governing that movement. For example, if $\mathrm{O}_{1}$ is a particular mutational state, then that which determines the probability of a prior mutational state, $\mathrm{B}_{1}$, transitioning to $\mathrm{O}_{1}$ are presumably facts relevant to random mutations (e.g. chemical facts, physical facts, etcetera). Collectively, these facts make it such that the transition probability of reaching $\mathrm{O}_{1}$ is 0.6 .

Now, suppose that only $A_{1}$ obtained. That is - it is, now, uncertain whether $B_{1}$, as opposed to another $\mathrm{B}$ event, will obtain. The probability of realising $\mathrm{O}_{1}$ would then be 0.18 (i.e. $0.3 * 0.6$ ). This makes intuitive sense as there is, now, greater uncertainty as to whether $B_{1}$ will occur: a different event, $\mathrm{B}_{(\mathrm{x})}$, may occur in lieu of $\mathrm{B}_{1}$, and then $\mathrm{O}_{1}$ would have failed to occur. Take even one step further back and consider the scenario whereby even $A_{1}$ had not yet obtained. In this case, the objective probability of $\mathrm{O}_{1}$ is 0.072 (i.e. $0.4 * 0.3 * 0.6$ ) since it is still an open question whether even $A_{1}$ will occur. $A_{1}$ 's objective probability is only 0.4 , after all. In fact, as the number of preceding events on which $\mathrm{O}_{1}$ is dependent upon increases (insofar as they are less than 1 probability), $\mathrm{O}_{1}{ }^{\prime} \mathrm{s}$ objective probability decreases. As we shall see in more detail, this relates to an adjustment of the

\footnotetext{
${ }^{25}$ As an aside, recognising the number of steps depends on the grain invoked; $B_{1}$ need not strictly be continuously preceding $\mathrm{O}_{1}$. That is - there may be a number of intermediary steps between $\mathrm{B}_{1}$ and $\mathrm{O}_{1}$. However, this complication does not affect the value of the transition probabilities, which is equivalent to the probability of $\mathrm{O}_{1}$ conditional upon $\mathrm{B}_{1}$.
} 
modal range. But, in brief, as we extend the modal range, there is greater opportunity for evolution to veer away from path(s) that realise $O_{1}$.

The single pathway picture presented above is simplified, for it does not take into account alternative sequences of events in which $\mathrm{O}_{1}$ could obtain. It is entirely possible that, in addition to the above causal pathway, there are two additional pathways that each consist of two preceding events. In this case, the total objective probability of $\mathrm{O}_{1}$ will be the products of the probability of each event in each individual causal pathway, summed together (as per Kolmogorov's axioms).

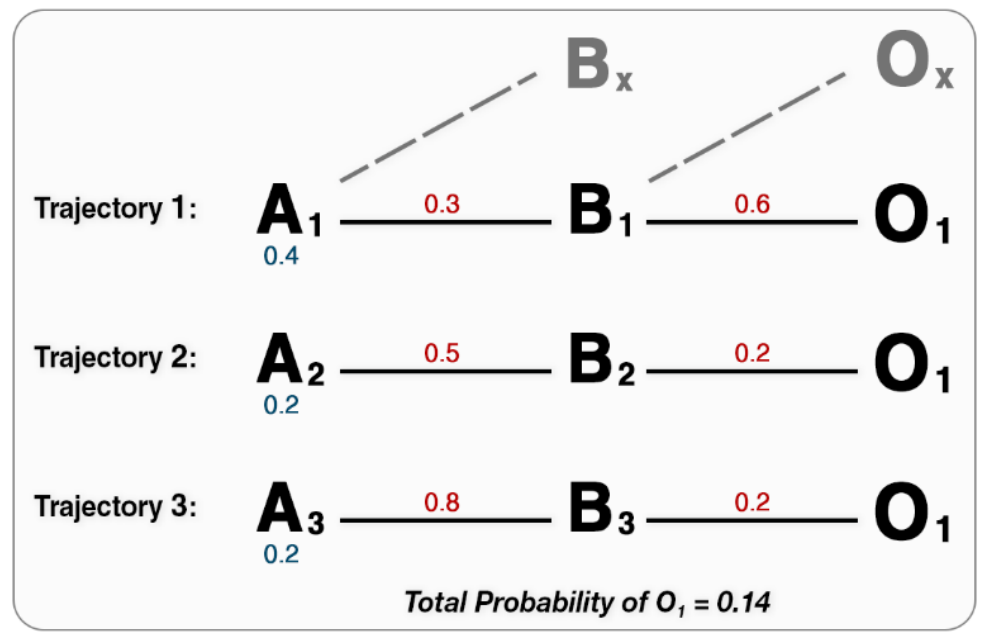

Figure 8. Multi-Causal Pathway to $\mathrm{O}_{1}$. (Assume that there are no other pathways to $\mathrm{O}_{1 .}$.)

So, whilst the evolution of $\mathrm{O}_{1}$ based only on trajectory 1 is 0.0072 , when all trajectories are considered, the total probability of evolution increases is 0.14 (as to respect the presence of any counterfactual robustness). The presence of alternative trajectories is, precisely, the second sort of modal component (i.e. counterfactual robustness) that evolutionary contingency ought to capture. This is especially crucial within the context of evolutionary biology whereby it is not uncommon for there to be more than one path for realising an evolutionary outcome. For example, on a molecular level, it has been demonstrated that there are multiple mutational pathways to glucose transport efficiency of $E$. coli (Manch et al., 1999).

Evolutionary contingency as non-trivial objective probability is also unifying in that it encompasses past proposals of contingency. That is - non-trivial objective probability of an outcome can be accounted for by some combination of etiological structures, if that should be the case. For example, an evolutionary outcome with a non-trivial objective probability can be produced in a system whereby there is sensitivity to initial conditions or path dependence insofar as there are low probability antecedents.

Relatedly, notice that evolutionary contingency as non-trivial objective probability is consistent with either of Gould's versions of the tape of life. Insofar as an outcome has a non-trivial objective probability, it is evolutionarily contingent whether an alteration of initial conditions would have produced a different outcome (replaying the tape of life from different conditions) or whether the same initial conditions would have produced a different outcome (replaying the tape of life from the same conditions). That is - evolutionary contingency as non-trivial objective probability incorporates both forms of modalities: trajectory propensity and counterfactual robustness. There is no need to quarrel about which version of the tape of life metaphor Gould really had in mind - both are consistent with evolutionary contingency as non-trivial objective probability. 
There has been a lingering question throughout: what does it mean, exactly, for an outcome to have a particular probability of evolution? The answer can be spelled out with the concept of the modal range (Wong, 2019).

Contingency claims are about the repeatability of certain biological forms across an array of evolutionary scenarios. But, barring some exceptions ${ }^{26}$, most contingency-theorists advocate that questions of evolutionary contingency are about the prevalence of biological forms amidst the variance of certain, important evolutionary conditions of epistemic interest. In other words, a biological form is non-contingent if it invariantly evolves in a wide range of evolutionary scenarios where (inter alia) the initial conditions, geographical space, developmental generators, history, or, even nomological laws (may) differ between scenarios. The range of evolutionary scenarios under consideration is called the modal range.

Modal Range: The Range of (Actual or Non-Actual) Evolutionary Scenarios, Under Consideration, in which an Outcome can Evolve

Accordingly, for any question of evolutionary contingency, what is being asked is whether the evolutionary dynamics within that modal range are sufficient to result in the repetitive (or probable) evolution of certain outcomes within that range. A different modal range index entails a different contingency question and is likely to result in a different answer.

Under the proposed probabilistic account, specifying the modal range is tantamount to specifying the reference class of a contingency claim. For instance, when it is said that a form has a specific probability of evolution, it is meant that the form evolves to that probability within a modal range. For any given outcome and modal range, if the probability of evolution is high, then the outcome is not very evolutionarily contingent (for the modal range). Conversely, for any given outcome and modal range, if the probability of evolution is low, then the form is evolutionarily contingent (for the modal range). It is when the proposed account is combined with the modal range that contingency claims are formulated in a conceptually precise way:

An outcome, $\mathrm{O}$, is evolutionarily contingent in modal range, $\mathrm{R}$, to the degree of objective probability, $\mathrm{P}$ (where $\mathrm{P}$ is in between 1 and 0 ).

This, then, presents a clear set of satisfaction conditions, which can be determined by some empirical means. Naturally, some modal ranges will offer high empirical tractability and others less so. Modal ranges that extend to nomologically inconsistent worlds or to the deep past may present difficulties for evaluation. Moreover, some modal ranges will be so narrow as to be uninteresting for evolutionary contingency. For example, whether some outcome evolves within a grossly restricted region of geographic space and time period tells us little about the general nomologies and historicities that one is ordinarily concerned with.

In general, the determination of the modal range is wholly dependent on the contingency-theorist's epistemic project and the nature of the evidence at hand (c.f. Wong, 2019). Relevant to its specification, the modal range can also differ according to the degree of variance amongst the range of evolutionary scenarios. If the modal range consists of differing evolutionary scenarios, then the

\footnotetext{
${ }^{26}$ For example, Beatty $(2016,2017)$ thinks that no initial differences need to exist between alternative replays of the tape of life in order for there to be different biological outcomes. Differences will naturally accumulate over time between 'replays' in an indeterminist universe. Contingency questions, then, can even be about the prevalence of form amongst a series of initially identical evolutionary scenarios. (If, however, evolutionary scenarios are coarse-grained enough to contain 'hidden variables', then such a position need not oppose causal determinism.) This also relates to parallel replay experiments (see later discussion).
} 
probability of the outcome's evolution speaks to the effect that those differences across scenarios impose. For example, if an outcome's probability of evolution turns out to be the same within two different modal ranges, where the first range contains evolutionary scenarios with an ostensibly important historical event and the second contains only evolutionary scenarios without that event, then it can be concluded that the historical event had no effect on the evolution of the outcome. In this case, the historical event had been made the independent variable which, as it turns out, made no difference to the outcome's probability of evolution. There is counterfactual robustness, so to speak. However, if the modal range consists of identical evolutionary scenarios, then the probability of an outcome's evolution speaks to only trajectory propensity. Some claims asserting contingency are made precisely when it is suspected that there are some independent variables, which do make a difference to a particular outcome's probability of evolution: e.g. 'the evolution of trait A is evolutionarily contingent because the necessary genetic precursor is not always possessed across species'. As we shall see later, experimenters (e.g. Blount, 2016; Blount et al., 2018) have manipulated the modal range, in this way, in order to derive certain conclusions from their studies.

In the remainder of this section, I shall consider evolutionary contingency as non-trivial objective probability in light of two additional desiderata.

\subsection{Varying in Degree}

In concert with my positive argument for evolutionary contingency as non-trivial objective probability, I shall argue that evolutionary contingency as non-trivial objective probability also satisfies the desideratum of varying in degree. In fact, it offers a single and straightforward means of fleshing out how contingency claims can differ by strength. Evolutionary outcomes are more or less contingent just in case the set of evolutionary outcomes are more or less probable within the modal range. Hence, if a certain evolutionary outcome has a high objective total probability of evolving within a modal range, then it is not very contingent. Conversely, if an outcome has a low total objective probability of evolving within the modal range, then it is highly contingent.

Contingency-theorists have consistently suggested that evolutionary contingency ought to be able to vary in degree (e.g. Beatty 2006; Desjardins, 2011a; Powell, 2012). This is driven by the normative consideration that the concept should map realistically onto the natural world where different evolutionary systems seemingly exhibit varying levels of contingency. Desjardins (2011a), for example, notes that, as per standard population genetics, the probability of evolving certain adaptive complexes is affected by the rate at which beneficial mutations occur $(\mu)$. But $\mu$, itself, is affected by population size $(\mathrm{N})$ which can vary drastically from population to population, and species to species. Small population sizes, with lower $\mu$, will have a lower probability of evolving certain adaptive complexes than larger population sizes with a higher $\mu$, ceteris paribus. For Desjardins (2011a), since population sizes vary widely in the natural world, it is also expected that different levels of contingency will be exhibited ${ }^{27}$. Similarly, population size also covaries with the strength of drift (Wright, 1931). Thus, if drift is taken to be a source of contingency, then there is yet another way for population size to influence the strength of contingency. Of course, none of this is to say that population size is the only way to affect the degree of contingency; rather, evolutionarily contingent dynamics are likely to be multi-variate and complex. The point is that population genetics suffice to stipulate that if $\mu$ and/or genetic drift affect the level of contingency, then evolutionary

\footnotetext{
${ }^{27}$ The implicit assumption is that contingency depends on $\mu$ and that the strength of contingency is not balanced out by other factors.
} 
contingency does indeed vary in degree. These normative considerations constitute as reasons for the first desideratum.

Now, if evolutionary contingency can vary in degree, then it is also spectral in the sense that there exists a range of views from the extreme to the intermediary (see Figure 9). Traditionally, contingency-theorists have, for convenience or otherwise, characterised the debate about evolutionary contingency in terms of two diametrically opposing views often dubbed the contingency thesis and the robust repeatability view or the robust view of life (RVL). Marking the opposition in this way is infelicitous as no actual biologist or contingency-theorist believes that any evolutionary system is entirely contingent or non-contingent. In reality, disagreements consist of opponents holding two intermediary views that are nonetheless inconsistent on account of their assertions of different levels of contingency.

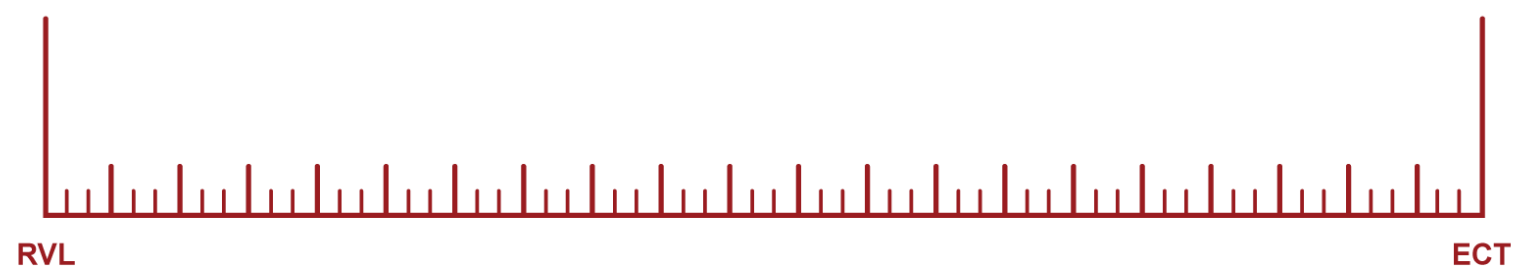

Figure 9. Contingency Spectrum (Wong, 2019)

The way in which evolutionary contingency is construed such as to be able to vary in degree depends on the sense of contingency invoked. However, evolutionary contingency as Beatty's (2006) causal dependence is strictly a binary notion: either an outcome is causally dependent on (i.e. covaries with) an antecedent or it is not.

In a recent paper, Lewis (2018) suggests that contingency is weaker or stronger depending on the relative 'volumes' of the set of initial conditions and outcomes. A volume, in Lewis' analysis, refers to the region of phase points in any hyperspace of possibility (e.g. a region in a morphospace). According to Lewis (2018), if a small volume of initial conditions leads to a large volume of outcomes, then contingency is said to be strong. This way of cashing out the way in which sensitivity to initial conditions varies in degree differs to that of Ben-Menahem (1997) and Powell (2012).

Ben-Menahem (1997) and Powell (2012) stipulate that the degree to which contingency varies is dependent only on the extent of differences between two sets of initial conditions. If slightly different initial conditions are sufficient to entail a different outcome, then the outcome is highly sensitive to initial conditions. If large differences in initial conditions are required before another outcome results, then the outcome of concern is lowly sensitive to initial conditions. The difference between the concerned outcome and the alternative outcome that would have resulted does not factor into the degree to which an outcome is sensitive to initial conditions. Ben-Menahem's (1997) and Powell's (2012) accounts are one-dimensional in this respect, whereas Lewis' account (2018) is two-dimensional.

Desjardins (2011a) suggests that path dependence can vary in degree according to three ways. Firstly, since path dependent processes admit of multiple outcomes, one can ask how similar the alternative outcomes are. If the outcomes are similar (according to some to-be-defined metric), then there is not much path dependence. The second concerns the number of paths that lead to one particular outcome. The lesser number of paths, the more path dependent an outcome is. The third way, and the central focus of Desjardins' two papers (2011a and 2011b), is how different the 
conditional probabilities of the possible outcomes are from each other ${ }^{28}$. For example, consider the branching diagrams of Figure 10, (a) and (b). According to Desjardins, the scenario illustrated in (a) is one of path dependence to a low degree because the conditional probabilities are nearly equitable whilst (b) is a scenario that is highly path dependent.

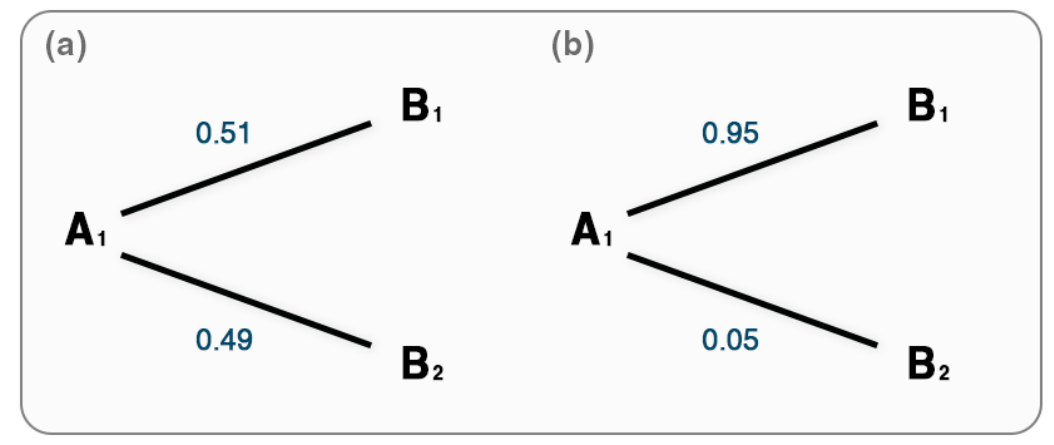

Figure 10. (a) Desjardins' Weak Path Dependence (b) Desjardins' Strong Path Dependence

With both sensitivity to initial conditions and path dependence, there is more than one way to construe how contingency varies in degree. It is not obvious that any one of these ways is superior. Perhaps, contingency is pluralistic with respect to the way in which it varies in degree, under these accounts. Ultimately, these two accounts nonetheless satisfy this desideratum unlike the causal dependence sense (see Table 1 at the end of the section). I shall now argue that the non-trivial objective probability also emphasises the importance of history.

\subsection{Subsumes Biological Historicities}

As discussed in section 3, the lesson from Wonderful Life (1989) is that history, itself, can be shaky.

Gould elaborated in his final and grand monograph, The Structure of Evolutionary Theory (2002), that history has a particular role to play in determining evolutionary outcomes. More specifically, the majority of historical evolutionary events "happened to happen in a certain particular way [my emphasis added]" (Ibid.) such that outcomes occurred in a certain way. The 'happened to happen' is telling here for it suggests that the historical event(s) in question need not have occurred - they have an objective probability of less than one. And yet, at the same time, downstream outcomes are significantly dependent on their occurrence. And so, for Gould, not only are some downstream outcomes dependent on historical events (i.e. causal dependence) but also that these historical events, themselves, may be uncertain.

In evolutionary biology, the conjunction of these two ideas has come to be known as historicities, first coined by Lewontin (1967), a close collaborator of Gould. Lewontin (1967) demonstrated that the dynamics of a population are affected by the probability distribution and ordering of various environmental features (Ibid.). These environmental features are what he considered to be historicities. Recent works, including Beatty and Desjardins (2009), Desjardins (2011a, 2011b, 2016), and Sterelny (2016), have redirected attention to the importance of biological historicities. It is argued, for instance, that historicities are the way in which outcomes are evolutionarily contingent (Beatty and Desjardins, 2009).

Historicities can be encapsulated by biological phenomena that are, indeed, quite central to modern evolutionary theory and were originally brought to bear by the so-called internalists (Gould, 1977;

\footnotetext{
${ }^{28}$ May I suggest that this is best quantitatively formalised with Shannon's (1948) information entropy; the larger the entropy, the greater the contingency.
} 
Gould and Lewontin, 1979). These phenomena do not so much as constitute important historical events on which future outcomes are hinged but, rather, they are the mechanisms by which future outcomes are constrained by certain low probability historical events. These phenomena include (but are not limited to) developmental constraints, phylogenetic inertia, and, genetic inheritance. They are united by the fact that they describe the constraining effects that individual events like random mutation, meiosis, or recombination have on evolving lineages; namely, a constraint on the possibility of downstream outcomes. For example, without the inheritance of the appropriate genetic material, offspring will simply not evolve certain traits. À la Krebs and Davies (1981), zebras cannot evolve machine guns with which to fend against predators, so to speak, due to their not inheriting the right genetic material (c.f. Shanahan, 2008).

But biological historicities extend beyond the genetic, developmental, or, phylogenetic. Factors such as asteroid impacts, mass extinctions, or geographic events can be rare occurrences that affect evolutionary outcomes. In the case of the Cambrian extinction (Gould, 1989), the series of sampling events that ultimately rendered the Pikaia a survivor, could have occurred differently, resulting in a different set of remaining forms. And hence, what matters is not only that there is causal dependence, but that the events themselves might not have occurred. It is the latter that precludes one version of the replaying the tape of life from apparently being a trivial exercise. On account of these historicities, the tape of life can be re-played, even without alteration, such that a different result obtains. After all, "[t]he divine tape player holds a million scenarios" (Gould, 1989). History is important, partly, because it is not certain ${ }^{29}$. Gould meant that evolution is contingent by way of the uncertainty of the past.

Evolutionary contingency as non-trivial objective probability gels well with such a picture. As mentioned earlier, objective probabilities of an outcome are dependent on the probability of preceding events, which, themselves, may have a probability of less than one (and more than zero). In other words, when those preceding events have a probability of less than one (and more than zero), they are uncertain. In contrast, evolutionary contingency as sensitivity to initial conditions, for example, does not subsume historicities. That is - the sensitivity of an outcome to its initial condition does not co-vary with the probability that certain initial conditions obtain. Even if the initial conditions had a high probability, as opposed to a low probability, the outcome would just be as sensitive to initial conditions. The reason is because what matters to sensitivity to initial conditions is whether a slightly different initial condition would have resulted in a markedly different outcome (Ben-Menahem 1997; Powell, 2012). On the other hand, path dependence (Type I and Type II) does subsume historicities as the probabilities of outcomes are a function of past events realised. Depending on which historical events, certain outcomes are made more or less probable; history is a difference maker.

There have been repeated efforts to recognise biological historicities ${ }^{30}$. For example, numerous authors have argued that the K-Pg asteroid drastically changed the environmental conditions of almost all species (e.g. Alvarez et al., 1980). One notable effect of the impact of the asteroid was the formation of dust clouds, which induced not only a global greenhouse effect, but greatly reduced the

\footnotetext{
${ }^{29}$ As a note aside, Gould took the uncertainty of evolutionary outcomes to be consistent with scientific enterprise. First, non-trivial probability events are taken to be nomologically consistent and explanatorily sound as supported by Gould's usage of "sensible channel", "each perfectly sensible" and "subject to rigorous explanation" (1989). Secondly, Gould claimed in Wonderful Life (1989), and elsewhere, that science itself is a study of history or that science requires historical narratives (idiographies).

${ }^{30}$ Orgogozo (2015) has impressively compiled a lengthy taxonomy of historicities, explicitly referring to the low probability nature of these events.
} 
number of photosynthesizing plants. The latter led to the decline and eventual extinction of dinosaurs. However, as the story goes, only when dinosaurs were out of the way, could mammals (including our ancestors) proliferate and expand. These significant downstream events are hinged on the asteroid impact, which is taken to be a rare occurrence.

There has been one particularly significant and well-documented study to which I wish to turn my focus: the Long Term Evolutionary Experiment (LTEE) by Blount et al. (2008). Twelve initially identical populations of $E$. coli, which did not have the ability to metabolise citrate for energy, were put on a glucose-limited medium that also contained citrate. At the 31,500 generation mark, one population (Cit+) miraculously evolved the ability to metabolise citrate, which enabled the population to explode in size. The new ability was taken to be a marvel due to certain chemical challenges of utilising citrate in oxic conditions (Koser et al., 1924; Lütgens \& Gottschalk, 1980), but one of Lenski's then graduate students, Blount, wondered whether such an evolutionary marvel was imminently repeatable or merely a fluke accident.

Fortunately, the ingenuity of the LTEE was also found in the study's design. At every 500 generations, samples from each of the twelve $E$. coli populations were frozen, thereby accumulating a historical record of E. coli samples and their genomes. Blount et al. (2008) employed this to their advantage and systematically thawed various generations of the ancestors of the Cit+ variant. They subsequently subjected a variety of these ancestors to the same environmental demands (i.e. high citrate, low glucose medium) to test for their ability to evolve the Cit+ variant. The results were interesting: they discovered that only certain ancestors - those after the 20,000-generation mark could repeatedly evolve the capacity to metabolise citrate. Importantly, however, ancestors before the 20,000 generation were unable to evolve the capacity to metabolise citrate even with a high number of trials. Given these two observations, Blount et al. (2008) reasoned that an important precursor allowing for citrate metabolism must have evolved (via random mutation) at around the 20,000 generation mark. But this mutation must also have been rare (or of low objective probability), since the same mutation failed to re-occur in any of the pre-20,000 generation ancestors.

In a follow up study, with the aid of DNA sequencing, Blount et al. (2012) confirmed this hypothesis and identified the relevant precursor gene that they dubbed citT. Although the molecular origin story of citT is complex (and, perhaps, this would speak to its rare occurrence), citT ultimately facilitated the eventual evolution of aerobic citrate utilisation through a simple duplication of citT and, subsequent, exaptation of it (Blount et al., 2012). In other words, the citT was just an evolutionary stone's throw away from citrate usage, yet cit $T$ was a rare mutational occurrence.

The moral of the story is that the evolution of citrate usage in the LTEE exemplifies a welldocumented historicity in that it fulfils two conditions: (i) it was a rare occurrence that need not have occurred and (ii) downstream outcomes are dependent on it. Clearly, a mutational event happened for one population of bacteria at around the 20,000 generation mark that did not occur in any of other initially identical populations. At the same time, once the cit $T$ was in place, the population was fast-tracked to the evolution of citrate usage. Gould's point in Wonderful Life (1989) was that evolution is littered with these historicities.

The manipulation of the frozen record of $E$. coli samples for controlled 'replays' also constitutes a case where there has been a clear adjustment of the modal range. Replays of $E$. coli samples at the 20,000 -generation mark constitute a narrower modal range than replays of samples before the 20,000-generation mark since the modal range of the former spans a shorter period of evolutionary time. What Blount et al. (2008) showed was that the proportion of lineages evolving citrate usage 
was higher in replays of more recent lineages (after, the 20,000 mark) than the proportion of lineages evolving citrate usage in replays in older lineages (i.e. 0 documented). In other words, there was less contingency in a narrower modal range but more contingency in a wider modal range. This was not surprising as the narrower modal range had the occurrence of the relevant historicity of low probability (i.e. the origination of the precursor, citT).

Relatedly, in a pair of recent review articles (Blount, 2016; Blount et al., 2018), the authors distinguished between 'Historical Difference Experiments' (HDE) and 'Parallel Replay Experiments (PRE). HDE's test for counterfactual robustness of outcomes whilst PRE's test for both counterfactual robustness and trajectory propensity. In Blount et al.'s (2008) study, the replays with the narrower modal range would have tested for only trajectory propensity (viz. even if the same historical precursor occurs, how often do we get the same outcome) whilst the replay with the wider modal range tested for both counterfactual robustness (viz. what if there had been different history) and trajectory propensity.

To summarise, the below table outlines how some of the previous accounts of evolutionary contingency fare with respect to the different desiderata presented in this paper:

\begin{tabular}{|c|c|c|c|c|c|}
\hline & $\begin{array}{c}\text { Causal } \\
\text { Dependence } \\
\text { (Beatty, 2006) }\end{array}$ & $\begin{array}{c}\text { Sensitivity to Initial } \\
\text { Conditions (Powell } \\
\text { \& Mariscal, 2012) }\end{array}$ & $\begin{array}{c}\text { Path Dependence } \\
\text { (Desjardins, } \\
\text { 2011a, 2011b, } \\
\text { 2016) }\end{array}$ & $\begin{array}{c}\text { Contingency Per } \\
\text { Se / New Causal } \\
\text { Unpredictability } \\
\text { (Beatty, 2016, } \\
2017)\end{array}$ & $\begin{array}{c}\text { Non-Trivial } \\
\text { Objective } \\
\text { Probability }\end{array}$ \\
\hline $\begin{array}{c}\text { Biological } \\
\text { Evitability }\end{array}$ & $\mathbf{X}$ & $\mathbf{X}$ & $\checkmark$ & $\checkmark$ & $\checkmark$ \\
\hline $\begin{array}{c}\text { Vary in } \\
\text { Degrees }\end{array}$ & $\mathbf{X}$ & $\checkmark$ & $\checkmark$ & $\checkmark$ & $\checkmark$ \\
\hline $\begin{array}{c}\text { Subsumes } \\
\text { Historicities }\end{array}$ & $\vee$ & $\mathbf{X}$ & $\checkmark$ & $\mathbf{X}$ & $\checkmark$ \\
\hline
\end{tabular}

Table 1. Evolutionary Contingency x Desiderata

However, as can be seen, only the path dependence account and non-trivial objective probability account satisfy all three desiderata. That is - on the basis of the desiderata, these two accounts fare equally just as well. There is, however, one notable point of difference. Although both path dependence and contingency as non-trivial objective probability subsume historicities, the two accounts do so in a different way. But, quite crucially for our purposes here, it is not clear that any one way is superior as to render one a better account of evolutionary contingency.

To see this, one needs to consider the original motivation for emphasising the importance of history in evolutionary biology. Gould and Lewontin (together and independently) intended to critique the adaptationist's ahistorical approach in evaluating optimality (Gould and Lewontin, 1979; Lewontin, 1967,1978 ). Their mission was to point out that adaptationism (methodological or otherwise) failed to be attuned to the fact that (i) the ancestral conditions (environmental or existing substrates with which evolution has to modify), (ii) whether or not these ancestral conditions obtain, and (ii) their ordering matter to whether a specific optimal complex is reached (Griffiths, 1996; c.f. Beatty and Desjardins, 2009). Both the path dependence account and non-trivial objective probability account are able to answer to these three elements

History is a difference maker in Desjardins' account; depending on which antecedent events occur, the probabilities of the outcomes are different. Consider the following example of path 
independence which fails Desjardins' third condition - a condition that was intended to help the account capture the effects of history $(2011,2011 b, 2016)$ :

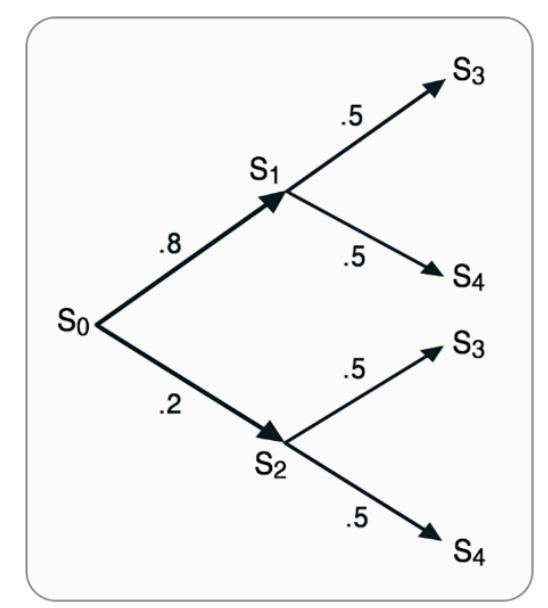

\section{Figure 11. Desjardins' Path Independence}

The events, $S_{3}$ and $S_{4}$, both have a conditional probability of 0.5 , which is conditional upon their respective immediately preceding events $\left(S_{1}\right.$ or $\left.S_{2}\right)$. However, both $S_{3}$ and $S_{4}$ are said to be outcomes at the end of a path independent chain because their conditional probabilities fail to change as a result of $S_{1}$ being realised or $S_{2}$ being realised. In other words:

$$
\operatorname{Pr}\left(S_{3} \mid S_{1}\right)=\operatorname{Pr}\left(S_{3} \mid S_{2}\right)
$$

and,

$$
\operatorname{Pr}\left(S_{4} \mid S_{1}\right)=\operatorname{Pr}\left(S_{4} \mid S_{2}\right)
$$

This fails the third of Desjardins' conditions for path dependence:

(Desjardins' Third Condition) The probabilities of the outcome changes as a function of the paths (or the sequence of previous events) realised ${ }^{31}$.

Thus, according to Desjardins, $\mathrm{S}_{3}$ and $\mathrm{S}_{4}$ are not outcomes that are path dependent (and hence, are not evolutionarily contingent outcomes despite there being evitability). Ultimately, the realisation of paths did not make a difference to the probabilities of $S_{3}$ or $S_{4}$. To be path dependent, as per the third condition, history ought to make a difference to how probable the final outcomes are.

However, even in the above path independence case, one could expect the path of ' $\mathrm{S}_{0}-\mathrm{S}_{1}-\mathrm{S}_{3}$ ' to be realised more often than ' $\mathrm{S}_{0}-\mathrm{S}_{2}-\mathrm{S}_{3}$ ' by a wide margin. This is because the conditional probability of $\mathrm{S}_{1}$ is 0.8 , whereas $S_{2}$ is only 0.2 . That is - whilst replaying the tape of life from $S_{0}$ for a number of trials would result in equal portions of $S_{3}$ and $S_{4}$ (barring any sampling error), the majority of the outcomes

\footnotetext{
${ }^{31}$ There is, however, a critical ambiguity in what it is that is to change as a function of previously realised events, which is not made clear in Desjardins' formulation of his third condition. Importantly, Desjardins' third condition is not referring to the total or unconditional probability of the outcome. In other words, it does not require that $\operatorname{Pr}\left(\mathrm{S}_{3}\right)$ or $\operatorname{Pr}\left(\mathrm{S}_{4}\right)$ change as a result of paths realised. It merely requires that $\operatorname{Pr}\left(\mathrm{S}_{3} \mid \mathrm{S}_{(\mathrm{x})}\right)$ change, which is to say that $\operatorname{Pr}\left(S_{3} \mid S_{1}\right) \neq \operatorname{Pr}\left(S_{3} \mid S_{2}\right)$.
} 
would have resulted from $S_{1}$ rather than $S_{2}$. As such, if $S_{1}$ or $S_{2}$ were able to stamp a mark of history on $S_{3}$ and/or $S_{4}$, then the process would also be information-preserving ${ }^{32}$.

The non-trivial objective probability account is also able to emphasise the importance of history in the three important respects suggested by Gould and Lewontin. As explained earlier, under the nontrivial objective probability account, historical events probabilistically contribute to an outcome's probability. In a sequence of antecedent events, the probabilities of these events determine the probability of an outcome. If the antecedents are of low probability, then the outcome occurring as a consequence of that sequence will be of low probability. But this is sufficient for the ancestral conditions, the 'chanciness' of ancestral conditions, and their ordering to matter to the outcome.

The ancestral conditions matter to the outcome in the sense that it makes an outcome more-or-less probable. The different sequences of events or trajectories will have different non-total, objective probabilities for an outcome. That is - an outcome may be highly probable under one sequence consisting of a specific string of historical events and lowly probable under another sequence consisting of a different string of historical events. Suppose that $A_{1}$ is a constituent of the former trajectory and, as such, helps make the former trajectory high probability whilst $A_{2}$ is of the latter trajectory and helps make the latter trajectory low probability. It follows that the antecedents $A_{1}$ and $A_{2}$ probabilistically contributed to the outcome differently. That is - although whether $A_{1}$ or $A_{2}$ occurred did not make a difference to the total objective probability of the outcome, $A_{1}$ and $A_{2}$ contributed differently to the total objective probability of the outcome. Perhaps, the $A_{1}$ added 0.4 to the total objective probability of the outcome whereas $A_{2}$ added only 0.02 . This differs from path dependence in that whether $A_{1}$ or $A_{2}$ is realised does not affect the total objective probability.

Secondly, it is clear that if the antecedent events have non-trivial objective probabilities, then the 'ancestral conditions' are uncertain. As per Gould, history "happened to happen in a certain particular way" (2002; my emphasis).

Thirdly, the effect of event ordering is built directly into sequence of probabilities. The order of events determines what the transition probabilities are in the sequence. Consider the below example, where there are two different ordered sequences and $\mathrm{G}_{1}$ and $\mathrm{G}_{2}$ refer to different mutational states. If $\mathrm{G}_{1}$ preceded $\mathrm{G}_{2}$, then the objective probability of $\mathrm{O}_{1}$ is 0.27 . This is because, for whatever reason, reaching $O_{1}$ from $G_{2}$ is easy. However, if $G_{2}$ preceded $G_{1}$, then the objective probability of $\mathrm{O}_{1}$ is only 0.1 . Event ordering (a) has a higher probability for $\mathrm{O}_{1}$ than event ordering (b). And, so the total objective probability of $O$ depends on whether a system has sequences with event ordering (a), event ordering (b), or both. In general, the order of events affects any outcome's total objective probability of evolution.

\footnotetext{
32 This depends on what the events $S_{3}$ and $S_{4}$ describe. If they are not exhaustive of system conditions, then they can be found with different correlates and differentiated as such. However, if all $S_{3} \mathrm{~S}$ are, ex hypothesi, identical under a system, then there can be no differentiating stamps.
} 


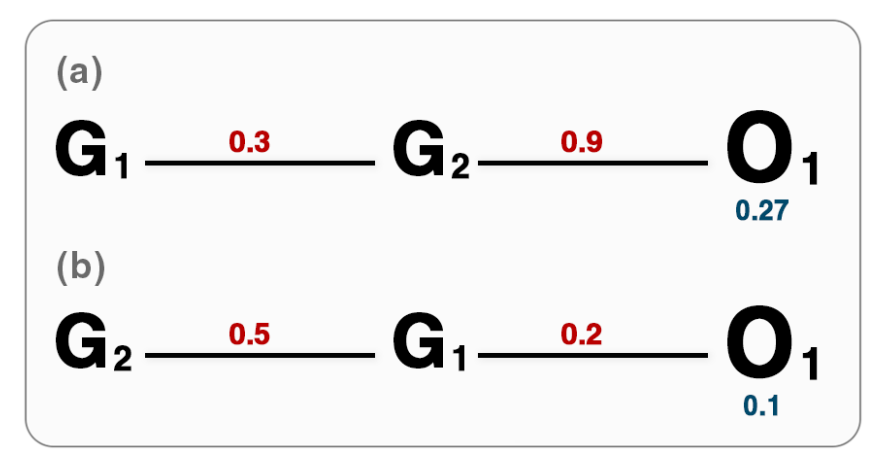

Figure 12. Event Ordering (a) and (b)

There is a commonality to evolutionary contingency as path dependence and evolutionary contingency non-trivial objective probabilities, which is that they both entail multiple possible outcomes. But Desjardins' account is stronger in that it also requires that the conditional probability concerned changes depending on which of the immediately preceding events is realised (i.e. his third condition). For this reason, under Desjardins' path dependence, history matters because they are difference makers to the outcome; the events that are realised make a difference to the probability that a given outcome occurs. However, for the non-trivial objective probability, history matters because antecedents play a part in determining how probable an outcome is to occur, but, at the same time, how probable an outcome is to occur also turns on the ordering of events. This is the primary difference between Desjardins' path dependence account and the present proposal of evolutionary contingency as non-trivial objective probability.

Despite this difference, it is not clear which is superior as to render one a better account of evolutionary contingency. Another point of difference between the two accounts is that the nontrivial objective probability is 'outcome-focused' whilst path dependence is 'process-dependent'. That is - the new account makes reference to only the properties of the outcome whilst the path dependence account is 'process-dependent' because, under path dependence, whether a particular outcome is evolutionarily contingent depends on the properties of possible paths to outcomes. Lacking a reason to favour being outcome-focused over process-dependent (or vice versa), this presents as no means for adjudication.

However, one way to adjudicate between the two accounts is to consider additional desiderata until a unique solution is reached, but this is a difficult task for, beyond the three desiderata hitherto presented, it is not clear what the other desiderata should be. And even if there are legitimately further desiderata, it may not distinguish between the two accounts if the both are equally satisfactory with respect to all the desiderata. For now, I leave it an open question and suggest that, perhaps, there is to be a sense of plurality in accounts of evolutionary contingency ${ }^{33}$.

\section{Conclusion}

Certainly, one additional desideratum might be empirical tractability (though this would seem to be for epistemic or practical convenience rather than theoretic reasons). The issue of empirical tractability has already been discussed at length with respect to the previous accounts of contingency (e.g. Beatty, 2006; Desjardins 2011b, 2015; Powell and Mariscal, 2015), where previous

\footnotetext{
${ }^{33}$ Independent to questions about the proper characterisation of contingency, etiological structures like sensitivity to initial conditions or path dependence are classes of dynamics that may be true for evolutionary systems - certainly, their appeal within the evolutionary contingency literature has been due to their plausibilities.
} 
proposals were satisfactory. The non-trivial objective probability account, however, is also empirically tractable.

To this end, I wish to conclude the paper by discussing some of the avenues in which the non-trivial objective probability account of contingency can be connected to the empirical evidence. Firstly, it should be said that the new account does not present any methodological difficulty beyond that of some previous accounts (i.e. radical contingency, path dependence) since these accounts already require probabilities to be determined. In fact, the novel account may be easier since path dependence, for example, requires, at least, three conditional probabilities to be determined before it can be said whether one outcome is path dependent.

On the whole, minimal welding is needed to connect evolutionary contingency as non-trivial objective probability with the evidence. This is because evolutionary biology is already full of tools and concepts that invoke probabilities. And, in some cases, the evidential connections are quite clear.

Take random mutations for example. It is common for mutational studies to chart, what is called mutational spectra, which indicate the frequencies that certain mutational outcomes obtain from an initial mutational state under study. Inherent in such a spectrum are probabilities, which can be easily calculated from the total number of mutational trials. That is - mutational spectra contain the information to determine the objective probabilities that certain mutational outcomes obtain from the initial mutational state and, as such, allow contingency-theorists to directly determine the contingency of certain mutational outcomes, albeit in quite narrow modal ranges as that of singular phase mutational transitions.

Laboratory experiments, including that of Blount et al.'s (2012), can also offer a means for the evaluation of probabilistic contingency claims. The successful origination of citT can be compared to the total number of evolutionary trials to calculate probabilities of its evolution. The LTEE, in particular, allows for an ingenious way of adjusting the modal range since there is a clear frozen record of all past states, ready for experimental manipulation.

In ecological contexts, the repeated appearance of a form across evolutionary scenarios (i.e. convergences) or its lack thereof (i.e. idiosyncrasies; c.f. Wong, 2019) can also be informative of evolutionary contingency as non-trivial objective probability. If a form is highly convergent across a modal range, then the probabilities of evolution are high, and the form is said to be non-contingent. In general, any study where probabilities of outcomes can be derived present an opportunity for the evaluation of evolutionary contingency as non-trivial objective probability. Evidence, however, need not come from probabilities computed from frequency testing.

Selection coefficients, which are inherently probabilistic, can also be invoked to analyse probabilistic contingency claims. One can, in theory, calculate a selection coefficient in a specific population and 'sum' these with any other factors that might impinge (e.g. other selection coefficients, genetic drift, migration flow) to determine the probability of the evolution of a trait in the population over a specific time frame (as specified by the models) - though it is easier said than done, owing to many idealisations and the difficulties of accurately determining all relevant evolutionary factors.

The proffered account of evolutionary contingency as non-trivial objective probability presents a world of opportunities for empirical tractability, beyond that which I can cover here. The objective probability of an outcome's evolution is but an extension of the nomologies, historicities, and so on, pertaining to a specific modal range. Yet, at the same time, these are the sorts of probabilities that 
can and/or have already been determined under a variety of scenarios with conceptual tools familiar to biologists.

Recently, (Beatty, 2016a, 2017) has stated that with evolutionary contingency, there should not be a need to rewind the tape of life and "alter" the initial conditions. One should merely rewind and play. This is true with the non-trivial objective probability sense. Under this account, rewinding to any point in the past of an evolutionarily contingent outcome, without an alteration, is (probably) sufficient for a different outcome to result on account of the fact that the sequence of preceding events was of non-trivial objective probability. The urge to avoid conflict with determinism has led to contingency-theorists, often, proposing etiologically heavy accounts of evolutionary contingency that fail, at least, one of the desiderata. But, in general, evolutionary contingency as non-trivial objective probability is unifying because it allows outcomes to have more or less contingency in any way that is relevant. It is unifying in the sense that it allows for the various proposals of continency to account for the non-trivial probability, if it should be the case. If an outcome is causally dependent on a preceding event but at the same time, that preceding event had low probability, then the evolutionary outcome has a non-trivial objective probability. Alternatively, an outcome can be sensitive to initial conditions such that there is no alternative trajectory that produces the outcome, but the total objective probability of evolution is going to be lower, ceteris paribus. Path dependence is also consistent with evolutionary contingency as low objective probability. Outcomes can be path dependent, but they can also have low objective probability.

Regardless of how evolutionarily contingent outcomes are produced, it may be said that an outcome, $\mathrm{O}$, is evolutionarily contingent in modal range, $\mathrm{R}$, to the degree of objective probability, $\mathrm{P}$ (where $\mathrm{P}$ is between 1 and 0 ). 


\section{Bibliography}

Arthur, B., 1989. Competing Technologies, Increasing Returns, and Lock-In by Historical Events. Economic Journal, 99, pp. 116-31.

Alvarez, L.W., Alvarez, W., Asaro, F., Michel, H.V., 1980. Extraterrestrial Cause for the CretaceousTertiary Extinction. Science, 208 (4448), pp. 1095-1108.

Baron, C., 2011. A web of controversies: Complexity in the Burgess Shale debate. Journal of the History of Biology, 44(4), pp. 745-780.

Beatty, J., 1995. The Evolutionary Contingency Thesis. In: Concepts, theories, and rationality in the biological sciences. University of Pittsburgh Press, Pittsburgh, pp. 45-81.

Beatty, J. and Carrera, I., 2011. When What Had to Happen Was Not Bound to Happen: History, Chance, Narrative, Evolution. Journal of the Philosophy of History 5(3), p. 471-495.

Beatty, J., 2006. Replaying life's tape. Journal of Philosophy, 103(7), pp.336-362.

Beatty, J., and Desjardins, E.C., 2009. Natural Selection and History. Biology and Philosophy, 24, pp. 231-46.

Beatty, J., 2016. What are Narratives Good for? Studies in History and Philosophy of Biological and Biomedical Sciences, 58, p. 33-40.

Beatty, J., 2017. Replaying Life's Tape - No Miracles Required. Extinct, [blog] 23 January. Available at: <http://www.extinctblog.org/extinct/2017/1/23/replaying-lifes-tape-no-miracles-required-1> [Accessed 21 January 2019]

Ben-Menahem, Y., 1997. Historical Contingency. Ratio, 10(2), pp. 99-107.

Ben-Menahem, Y., 2009. Historical Necessity and Contingency. In: A Companion to the Philosophy of History and Historiography. Blackwell Publishing Ltd.

Blount, Z. D., Borland, C. Z., Lenski, R. E., 2008. Historical contingency and the evolution of a key innovation in an experimental population of Escherichia coli. Proceedings of the National Academy of Sciences of the United States of America, 105, p. 7899-7906

Blount, Z.D., Barrick, J.E., Davidson, C.J., Lenski, R.E., 2012. Genomic analysis of a key innovation in an experimental Escherichia coli population. Nature, 89(7417), pp. 513-518.

Blount, Z.D., 2016. History's Windings in a Flask: Microbial Experiments into Evolutionary Contingency. In Ramsey G and Pence C (ed.) Chance and Evolution. University of Chicago Press, Chicago.

Blount Z.D., Lenski R.E., Losos J.B., 2018. Contingency and Determinism in Evolution: 'Replaying the Tape of Life'. Science, 362(6415):eaam5979

Conway Morris, S., 1998. The Crucible of Creation: The Burgess Shale and the Rise of Animals. New York: Oxford University Press.

Conway Morris, S., 2003. Life's solution: Inevitable humans in a lonely universe. Cambridge:

Cambridge University Press.

David, A. 2001. Path dependence, its critics and the quest for "historical economics". In: Evolution and path dependence in economic ideas: past and present. Elgar Publishing, Cheltenham. 
Desjardins, E., 2011a. Reflections on Path Dependence and Irreversibility: Lessons from Evolutionary Biology. Philosophy of Science, 78 (5), p. 724-738.

Desjardin, E., 2011b. Historicity and Experimental Evolution. Biology \& Philosophy, 26, p. 339- 64.

Desjardins, E., 2015. Historicity and Ecological Restoration. Biology \& Philosophy, 30, pp. 77-98.

Desjardin, E., 2016. Contingent Evolution: Not By Chance Alone. In Ramsey, G., \& Pence, C. eds, Chance in Evolution. University of Chicago Press.

Earman, J., 1986. A Primer on Determinism. Springer Netherlands.

Erwin, D. H., 2016. Wonderful life revisited: chance and contingency in the Ediacaran- Cambrian radiation. In Ramsey, G. and Pence, C. H., editors, Chance in evolution. University of Chicago Press, Chicago

Ferus, M., et al., 2015. High-energy chemistry of formamide: $A$ unified mechanism of nucleobase formation. Proceedings of the National Academy of Sciences USA, 112, pp. 657-662.

Ferus, M., et al., 2017. Formation of nucleobases in a Miller-Urey reducing atmosphere. Proceedings of the National Academy of Sciences USA, 114, pp. 4306-4311.

Gibbs, J.W., 1902. Elementary Principles of Statistical Mechanics. Yale University Press.

Gould, S.J. and Lewontin, R.C., 1979. The Spandrels of San Marco and the Panglossian Paradigm: a critique of the adaptationist programme. Proceedings of the Royal Society, London, 205(1161), pp. 581- 598 .

Gould, S. J., 1976. The genomic metronome as a null hypothesis. Paleobiology, 2(2), pp. 177-179.

Gould, S.J., 1977. Ontogeny and Phylogeny. Harvard University Press.

Gould, S. J., 1989. Wonderful life: The Burgess Shale and the nature of history. New York: Norton.

Gould, S.J., 1979. Letter to Schopf. 25 June 1979.

Gould, S. J., 2002. The structure of evolutionary theory. Cambridge: Harvard University Press.

Griffiths, P.E., 1996. The historical turn in the study of adaptation. British Journal for the Philosophy of Science, 47, pp. 511-532.

Krebs, J. R. and Davies, N. (1981). An introduction to behavioural ecology. Sinauer Associates, Sunderland, MA.

Koser, S.A., 1924. Correlation of Citrate Utilization by Members of the Colon-Aerogenes Group with Other Differential Characteristics and with Habitat. Journal of Bacteriology, 9, pp. 59-77.

Lewis, C.T., 2018. The Domain Relativity of Evolutionary Contingency. Biology \& Philosophy, 33 (3-4), pp. 25-43.

Lewis, D. 1973. Counterfactuals. Oxford: Blackwell.

Lewis, D. 1986. Philosophical Papers: Volume II. Oxford: Oxford University Press.

Lewontin, R.C., 1967. The principle of historicity in evolution. In: Moorhead PS, Kaplan MM (eds) Mathematical challenges to the neo-Darwinian interpretation of evolution. The Wistar Institute Press, Philadelphia, pp 81-88 
Lewontin, R.C., 1978. Adaptation. Scientific American, 239(3), pp. 156-169

Lewontin, R.C., 1983. The Organism as Subject and Object of Evolution. Scientia, vol. 188, pp. 65-82.

List, C., \& Pivato, M., 2015. Emergent chance. Philosophical Review, 124 (1), p. 119-152.

Lütgens, M., \& Gottschalk, G., 1980. Why a co-substrate is required for anaerobic growth of Escherichia coli on citrate. Journal of Genetic Microbiology, 119(1), pp. 63-70.

Liebowitz, S., and Margolis, S., 1995. Path Dependence, Lock-in, and History. Journal of Law, Economics and Organization, 11 (1), pp. 205-226.

Manche, K., Notley-McRobb, L., Ferenci, T., 1999. Mutational adaptation of Escherichia coli to glucose limitation involves distinct evolutionary pathways in aerobic and oxygen-limited environments. Genetics, 153, pp. 5-12

McConwell, A., and Currie, A. 2016. Gouldian arguments and the sources of contingency. Biology and Philosophy, 32 (2), 243-261.

Millstein, Roberta L., 2011. Chances and Causes in Evolutionary Biology: How Many Chances Become One Chance. In McKay Illari, P., Russo F., and Williamson, J., eds. Causality in the Sciences. Oxford University Press, p. 425-444.

Orgogozo, V., 2015. Replaying the tape of life in the twenty-first century. Interface Focus, 5(6):20150057.

Powell, R., 2012. Convergent Evolution and the Limits of Natural Selection. European Journal of the Philosophy of Science, 2(3), pp. 355-373.

Powell, R., and Mariscal, C. 2015. Convergent evolution as natural experiment. Interface Focus.

Saladino, R., Crestini, C., Costanzo, G., Negri, R., Di Mauro, E., 2001. A possible prebiotic synthesis of purine, adenine, cytosine, and 4(3H)-pyrimidinone from formamide: Implications for the origin of life. Bioorganic \& Medicinal Chemistry, 9, pp. 1249-1253.

Shanahan, T., 2008. Why don't zebras have machine guns? Adaptation, selection, and constraints in evolutionary theory. Studies in History and Philosophy of Biological and Biomedical Sciences, 39(1), pp. 135-46.

Shannon, C. E., 1948. A mathematical theory of communication. Bell System Technical Journal, 27(3), pp. 379-423.

Sterelny, K., 2005. Another view of life. Studies in History and Philosophy of Biological and Biomedical Sciences, 36, pp. 585-593.

Sterelny, K., 2016. Contingency and History. Philosophy of Science, 83(4), p. 521-539.

Sober, E., 1988. Reconstructing the past: parsimony, evolution, and inference. MIT Press, Cambridge. Turner, D., 2011a. Gould's replay revisited. Biology and Philosophy, 26, p.65-79.

Turner, D, 2011b. Paleontology: A Philosophical Introduction. Cambridge University Press.

Waddington, C.H., 1939. An Introduction to Modern Genetics. George Allen \& Unwin Ltd, London.

Waddington, C.H., 1940. Organisers and Genes. Cambridge University Press, Cambridge. 
Waddington, C.H., 1957. The Strategy of the Genes. George Allen \& Unwin Ltd, London.

Wright, S., 1931. Statistical theory of evolution. Journal of the American Statistical Association, 26(173A), pp. 201-208.

Wong, T.Y.W, 2019. The evolutionary contingency thesis and evolutionary idiosyncrasies. Biology \& Philosophy, 34:22. 